\title{
Commensal-derived metabolites govern Vibrio cholerae pathogenesis in host intestine
}

\author{
Jin Sun You ${ }^{1,2+}$, Ji Hyun Yong ${ }^{1,2 \dagger}$, Gwang Hee Kim², ${ }^{1,2}$ Sungmin Moon ${ }^{2,3}$, Ki Taek Nam², Ji Hwan Ryu ${ }^{2,3}$,
} Mi Young Yoon ${ }^{1,2,4^{*}}$ and Sang Sun Yoon ${ }^{1,2,4^{*}}$ (i)

\begin{abstract}
Background: Recent evidence suggests that the commensal microbes act as a barrier against invading pathogens and enteric infections are the consequences of multi-layered interactions among commensals, pathogens, and the host intestinal tissue. However, it remains unclear how perturbations of the gut microbiota compromise host infection resistance, especially through changes at species and metabolite levels.

Results: Here, we illustrate how Bacteroides vulgatus, a dominant species of the Bacteroidetes phylum in mouse intestine, suppresses infection by Vibrio cholerae, an important human pathogen. Clindamycin $(\mathrm{CL})$ is an antibiotic that selectively kills anaerobic bacteria, and accordingly Bacteroidetes are completely eradicated from CL-treated mouse intestines. The Bacteroidetes-depleted adult mice developed severe cholera-like symptoms, when infected with $V$. cholerae. Germ-free mice mono-associated with $B$. vulgatus became resistant to $V$. cholerae infection. Levels of $V$. cholerae growth-inhibitory metabolites including short-chain fatty acids plummeted upon $\mathrm{CL}$ treatment, while levels of compounds that enhance $V$. cholerae proliferation were elevated. Furthermore, the intestinal colonization process of $V$. cholerae was well-simulated in CL-treated adult mice.

Conclusions: Overall, we provide insights into how a symbiotic microbe and a pathogenic intruder interact inside host intestine. We identified B. vulgatus as an indigenous microbial species that can suppress intestinal infection. Our results also demonstrate that commensal-derived metabolites are a critical determinant for host resistance against $V$. cholerae infection, and that $C L$ pretreatment of adult mice generates a simple yet useful model of cholera infection.
\end{abstract}

Keywords: Vibrio cholerae, Gut microbiota, Colonization resistance, Short-chain fatty acids, Metabolomics, Bacteroides vulgatus, Clindamycin, Amino sugars

\section{Background}

Vibrio cholerae is the causative agent of pandemic diarrheal disease, cholera. While cholera toxin (CT) and toxincoregulated pilus (TCP) are known to be the major virulence determinants, its pathogenic mechanisms are starting to be understood as consequences of interaction with indigenous microbes, collectively termed gut microbiota [1-4]. A key feature of the gut microbiota is its protective capacity against enteropathogenic

\footnotetext{
* Correspondence: pepogo@yuhs.ac; sangsun_yoon@yuhs.ac

${ }^{\dagger}$ Jin Sun You and Ji Hyun Yong contributed equally to this work.

'Department of Microbiology and Immunology, Yonsei University College of

Medicine, 50-1 Yonsei-ro, Seodaemun-gu Seoul, Seoul 03722, Korea
}

Full list of author information is available at the end of the article infections, termed "colonization resistance" [5-7]. This property can be ascribed to the microbial ecosystem that is formed within the host intestine. This microbial ecosystem is an ever-changing community of various microbial species regulated by a complex network of microbiota-intrinsic and microbiota-extrinsic factors [8].

Microbiota-intrinsic factors such as interactions between the gut-residing species serve as the primary determinant of community composition. Such interactions include interbacterial niche competition [9] and secretion of antimicrobial substances [10]. For example, Bacteroides species that reside in the human gut, such as Bacteroides fragilis and Bacteroides uniformis, utilise

(c) The Author(s). 2019 Open Access This article is distributed under the terms of the Creative Commons Attribution 4.0 International License (http://creativecommons.org/licenses/by/4.0/), which permits unrestricted use, distribution, and reproduction in any medium, provided you give appropriate credit to the original author(s) and the source, provide a link to the Creative Commons license, and indicate if changes were made. The Creative Commons Public Domain Dedication waiver (http://creativecommons.org/publicdomain/zero/1.0/) applies to the data made available in this article, unless otherwise stated. 
membrane attack complex/perforin toxins BSAP-1 and BSAP-2 for intraspecies antagonism [11]. Microbiota-extrinsic factors include inflammation, diet, and antibiotic treatment. However, the aforementioned microbiota-intrinsic and extrinsic factors do not operate in exclusion of another. For example, compositional shift induced by an external factor may, in turn, modulate the host immune response via production of specific metabolites [8].

Recent studies have demonstrated that antibiotic treatments alter the gut microbiota in humans and other mammals [12-15], and increase the susceptibility of the host to infections by various enteric pathogens such as Shigella flexneri [16], Salmonella enterica [17], Clostridium difficile $[18,19]$, and vancomycin-resistant Enterococcus [20]. The abolishing effect of antibiotic treatment on host resistance to infection is most likely implemented through a multitude of factors, including suppression of specific microbial species, alteration of the metabolomic landscape as a result of the changed microbiome composition, and/or host responses [21-23]. These findings prompted us to investigate the effects of different classes of antibiotics on the gut microbiota and how they relate to host resistance against $V$. cholerae infection.

In this study, we uncover a unique microbiota-extrinsic treatment that induces severe cholera-like symptoms in adult mice, that are otherwise completely resistant. Furthermore, we show that a dramatic shift in metabolome production profile accounts for the compromised infection resistance in the host. This report highlights the importance of commensal-derived metabolites as a crucial determinant of host susceptibility to enteric infection.

\section{Results}

Clindamycin-treated adult mice exhibited dramatic gut microbiota compositional changes and became susceptible to $V$. cholerae infection

Colonization of enteric pathogens occurs depending on the composition of indigenous microbes inside the host intestine $[5,24,25]$. In order to observe the effects of gut microbiota compositional changes on host infection resistance under diverse experimental conditions, we treated adult C57BL/6 specific pathogen-free (SPF) mice (8 weeks of age) with three different antibiotics that have a distinct mode of bacterial killing; streptomycin (SM), vancomycin (VAN), and clindamycin (CL). SM is a broad-spectrum antibiotic that targets both Gram-positive and Gram-negative bacteria. VAN is an effective antibiotic against Gram-positives [26], while CL is known to selectively kill anaerobes $[27,28]$. After daily treatment for 5 days, mouse feces were collected to analyze microbiota composition by $16 \mathrm{~S}$ rRNA gene sequencing. Five bacterial phyla were found to have characteristic distributions among groups (Fig. 1a-d). In the untreated control group, Bacteroidetes was the most abundant phylum and the members of that single phylum occupied approximately $80 \%$ of the entire microbiota population (Fig. 1a). Upon SM treatment, the Verrucomicrobia phylum emerged, while the Bacteroidetes phylum maintained its predominant occupancy (Fig. 1b). VAN treatment resulted in multiplication of bacterial cells belonging to the Verrucomicrobia and Proteobacteria phyla, both of which are Gram-negatives (Fig. 1c). As a result of CL treatment, the Bacteroidetes phylum was eradicated, while the Firmicutes phylum remained largely unchanged in number (Fig. 1d). This observation is consistent with previous findings that $\mathrm{CL}$ is effective in treating infections of Bacteroides fragilis, a major species of the Bacteroidetes phylum [29, 30]. On the other hand, the Proteobacteria phylum underwent explosive expansion during $\mathrm{CL}$ treatment, further demonstrating that CL is not effective in inhibiting the growth of facultative anaerobes (Fig. 1d).

Subsequently, the mice of each antibiotic group were infected with $5 \times 10^{8} \mathrm{CFU}$ of the $V$. cholerae 7 th pandemic strain, N16961, to monitor susceptibility to infection and infection-induced microbiota composition changes. In the control and SM-treated groups, microbiota composition did not change much following $V$. cholerae infection (Fig. 1e, f). In response to N16961 infection, the relative abundance of the Bacteroidetes phylum increased in the VAN-pretreated group (Fig. $1 \mathrm{~g}$ vs. c). In CL-treated mice, the relative abundance of the Firmicutes phylum increased, while that of the Proteobacteria phylum decreased in response to $V$. cholerae infection (Fig. 1h). Importantly, among the Proteobacteria phylum detected in Fig. 1h, $\sim 8.81 \%$ was found to be derived from $V$. cholerae-specific $16 \mathrm{~S}$ rRNA gene sequence (Fig. 3). This result is worthy of particular attention because $V$. cholerae-specific sequence was not detected in the Proteobacteria populations in other groups (Fig. 1f, g).

Our results indicate that $V$. cholerae may actively colonize the adult mouse intestine under conditions created by CL treatment. To gain more insight into the outcomes of $V$. cholerae infection, we investigated whether any anatomical change was induced in the gastrointestinal tract ranging from the small intestine to the rectum. In the first three experimental groups, mouse tissues appeared similar to one another and no major changes were observed following $V$. cholerae infection (Fig. 1i-k). In contrast, infection-induced phenotypes were clearly demonstrated in the CL-treated group for the following indications (Fig. 11). First, fluid accumulation was clearly observed in the small intestine (red arrowheads). Second, the size of the cecum 


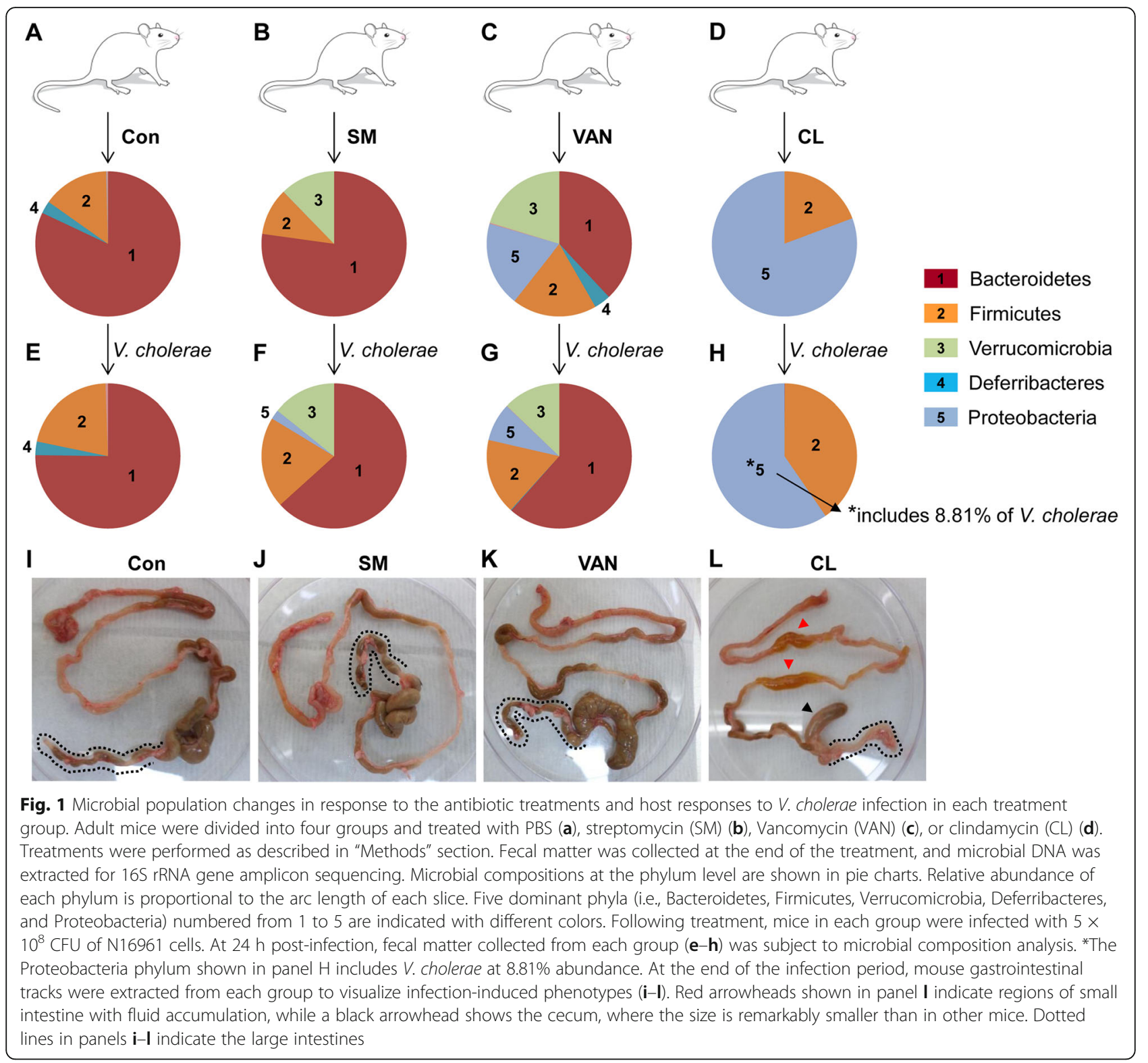

was markedly smaller compared to those in other groups (black arrowhead). Third, the colon appeared shorter and more interestingly, became transparent, while the colons in other groups were filled with feces (dotted line around the colon). Forth, fecal matter discharged from the CL-treated group was highly aqueous (data not shown). Together, these results demonstrate that $V$. cholerae infection induces cholera-like symptoms in adult mice, when their gut microbiota compositions are altered by CL treatment.

\section{$\mathrm{CL}$ treatment with a different regimen similarly induced hypersusceptibility to $V$. cholerae infection}

Our results, shown in Fig. 1, demonstrate that symptoms of watery diarrhea observed in human cholera patients can also be induced in adult mice by a simple antibiotic pretreatment. In the above experiment, mice were treated with a mild concentration of CL daily for 5 days. To elucidate whether CL treatment could elicit similar effects within a shorter period, we conducted another set of infection experiments with a single treatment of higher CL dosage (Additional file 1: Fig. S1A). Similar to previous results with lower CL dosage, significantly increased fluid accumulation (FA) was induced in adult mouse intestine at $24 \mathrm{~h}$ post-infection. The FA ratio was determined to be $\sim 0.176$, a value $\sim$ 1.6-fold higher in comparison to that of the uninfected group (Additional file 1: Fig. S1B). Consistent with this finding, robust $V$. cholerae colonization was observed in CL-pretreated mice. The number of $V$. cholerae cells 
recovered from the small intestine (SI) or cecum was remarkably larger in CL-treated vs. control group. $V$. cholerae cells of $>10^{9} \mathrm{CFU}$ were grown per gram sample in SI and cecum, respectively. Likewise, $\sim 10^{9} \mathrm{CFU}$ was also detected in fecal pellets shed from CL-treated mice (Additional file 1: Fig. S1C). In contrast, V. cholerae cells were not detected at all in feces discharged from the control group. Given that an infection dosage of $5 \times 10^{8} \mathrm{CFU}$ was used, these results demonstrate that (i) V. cholerae cells were almost completely eliminated inside the intestine of regular adult mice while (ii) the same number of inoculated cells was more highly proliferated in the CL-pretreated adult mouse intestine.

\section{Germ-free mice transplanted with feces of CL-treated mice were susceptible to $V$. cholerae infection}

Previous studies have revealed that CL treatment can modulate the production of pro-inflammatory cytokines, especially in response to lipopolysaccharide [31-33]. Furthermore, CL at sub-lethal concentrations stimulate neutrophil phagocytosis in vitro against Staphylococcus aureus [34] and Bacteroides spp. [35]. While our results strongly suggest that $\mathrm{CL}$-induced changes in gut microbiota composition are associated with elevated susceptibility to $V$. cholerae infection, these previous reports also indicate that enhanced $V$. cholerae infectivity in adult mice might be attributed to CL-induced modulation of the host immune response. To address this issue, we conducted fecal microbiota transplantation (FMT) to germ-free (GF) mice and tested whether the GF mice with reconstituted intestinal microbiota develop differential resistance to $V$. cholerae infection. Fecal pellets freshly collected from PBS- or CL-treated mice were resuspended in PBS and transferred to GF mice via oral gavage. Following a 3day transplant stabilization period, $5 \times 10^{8} \mathrm{~N} 16961$ cells were infected (Fig. 2a). GF mice transplanted with PBS-treated control fecal pellets developed strong resistance to the infection. Viable $V$. cholerae was recovered neither from the SI of all six mice nor from the cecum isolated from five out of six mice (Fig. 2b). While varying degrees of N16961 cells were detected in feces discharged from the GF mice transplanted
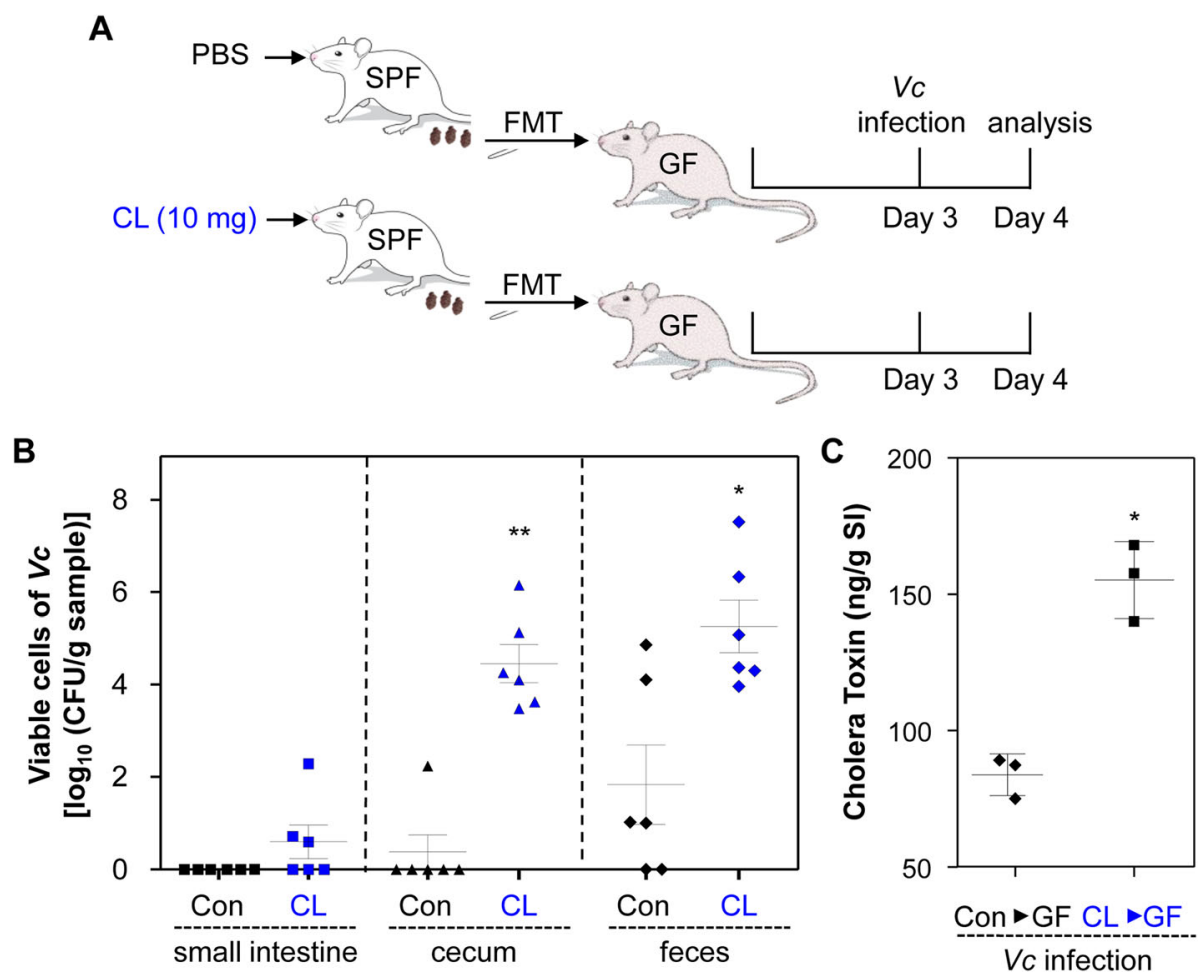

Fig. 2 Germ-free mice transplanted with feces of CL-treated mice are susceptible to $V$. cholerae infection. a Schematic diagram of the experimental procedure. Germ-free mice (C57BL/6, 8 9 weeks old, $n=6$ per group) received fecal suspensions (50 $\mu \mathrm{L})$ derived from PBS- or CLtreated SPF mice. Following 3 days of stabilization, transplanted mice were challenged with V. cholerae infection $\left(5 \times 10^{8} \mathrm{CFU}\right)$ for $24 \mathrm{~h}$. b The SI and cecum were removed from each mouse for tissue homogenization. Fresh fecal matter was collected to prepare fecal suspensions. Aliquots of lysates or suspensions were serially diluted to count CFU of $V$. cholerae on LB agar supplemented with $200 \mu \mathrm{g} / \mathrm{mL}$ SM. Values are displayed on a log scale as mean \pm SEM for each group. ${ }^{*} P<0.05$ versus bacterial CFU detected in the control group. ${ }^{* *} P<0.001$ versus bacterial CFUs detected in the control group. $\mathbf{c}$ In a separate set of experiments ( $n=3$ per group), small intestines were extracted for tissue homogenization, and aliquots of homogenates were assessed for detection of $C T$ by ELISA. ${ }^{*} P<0.01$ versus $C T$ level in the control group 
with control feces, four out of six mice produced feces that contained ten or fewer N16961 cells per gram (Fig. 2b). In contrast, N16961 colonization occurred more actively in the GF mice transplanted with CLtreated and therefore Bacteroidetes-depleted feces. In cecum and feces, $>10^{4}$-fold and $>10^{3.4}$-fold higher bacterial colonization were observed, respectively, compared with the control group (Fig. 2b).

We next asked whether production of CT, a critical virulence determinant, was accordingly increased during active $V$. cholerae colonization. As shown in Fig. 2c, in response to $V$. cholerae infection, CT production was significantly increased in mice transplanted with fecal suspensions of CL-treated mice. Since CL would not be much left in the feces discharged from the CL-treated mice, our results demonstrate that altered gut microbiota composition induced by $\mathrm{CL}$ treatment is directly responsible for rendering the murine host more susceptible to $V$. cholerae infection.

\section{Bacteroides vulgatus is the predominant species in the Bacteroidetes phylum and it inhibited $V$. cholerae growth in vivo}

Based on our results presented in Fig. 1, elevated $V$. cholerae infection in CL-pretreated mice could be ascribed either to the depletion of the Bacteroidetes or to the expansion of Proteobacteria phylum. Meanwhile, we noticed the followings. First, the Proteobacteria population similarly sufficiently propagated in VANtreated mice, a group that did not develop cholera-like symptoms (Fig. 1c). Second, the relative abundance of the Bacteroidetes phylum increased from $~ 38 \%$ (before infection) to $\sim 61 \%$ (after infection) in VANtreated mice (Fig. 1c, g). Based on these results, we postulated that the lack of cholera-like symptoms in the VAN-treated group was due not only to the presence of the Bacteroidetes phylum but also to the infection-induced expansion of its population. We hypothesized that the Bacteroidetes phylum, which predominantly occupies the adult mouse intestine, plays a critical role in protecting its host from $V$. cholerae infection.

To provide an insight into which bacterial species are core members of the Bacteroidetes phylum, we performed a species-level population analysis using $16 \mathrm{~S}$ rRNA gene sequencing. When necessary, we conducted PCR reactions with species-specific primer sets and verified the sequences of amplification products (data not shown). Three distinct bacterial species were determined to belong to the Bacteroidetes phylum; Bacteroides vulgatus, Parabacteroides goldsteinii, and Bacteroides caccae. In control mice, B. vulgatus was found to be present in the largest quantity, with a relative abundance greater than $50 \%$ of the entire population (Fig. 3). In response to VAN treatment, $P$. goldsteinii propagated, whereas B. vulgatus was diminished in population size. Of note, however, the relative abundance of the $B$. vulgatus population was recovered to its original level following $V$. cholerae infection. P. goldsteinii has been reclassified from Bacteroides goldsteinii [36] and a strain isolated from human intestine was resistant to VAN treatment [37]. As shown in Fig. 1d, l, no $16 \mathrm{~S}$ rRNA gene sequences matching any of these three species were detected in feces discharged from CL-treated mice either before or after $V$. cholerae infection (Fig. 3).

Because $B$. vulgatus was the most dominant species in the adult mouse intestine, we next assessed, under a more defined condition, the effect of B. vulgatus on altering host susceptibility to $V$. cholerae infection. To this end, we mono-associated GF mice with a suspension of B. vulgatus cells, grown anaerobically in vitro (Fig. 4a). B. vulgatus colonies cultivated straight from mouse fecal suspensions were subject to RAPD assay and an identical amplification pattern was observed between isolated colonies, thereby leading us to conclude that most colonies were clonal (Additional file 2: Figure S2). As shown in Fig. 4a, GF mice were also mono-associated with heat-killed B. vulgatus before being infected with $V$. cholerae cells as an additional negative control. At $24 \mathrm{~h}$ post-infection, significantly reduced numbers of $V$. cholerae cells were recovered in animals that were mono-associated with live $B$. vulgatus. In SI and colon, 830-fold and $\sim 40$-fold less $V$. cholerae colonization were observed, respectively, as compared with the PBS control group. Likewise, $V$. cholerae cell numbers, recovered from feces of the GF mice transplanted with live $B$. vulgatus, was $\sim 75$-fold lower than the PBS control (Fig. 4b). Importantly, $V$. cholerae colonization was never decreased in the animals mono-associated with heat-inactivated $B$. vulgatus cells (Fig. 4b). These results suggest that active $B$. vulgatus cells, when present in adult mouse intestine, can suppress $V$. cholerae colonization.

We next explored whether $B$. vulgatus also effectively inhibits $V$. cholerae colonization in the infant mouse model that has been used to study $V$. cholerae infection $[38,39]$. Five-day-old infant mice were divided into two groups, with the first group being transplanted with $B$. vulgatus and the other group serving as a negative control (Fig. 4c). B. vulgatus, when transplanted in the intestine of the infant mouse, can also effectively suppress $V$. cholerae colonization and fluid accumulation (Fig. 4d). V. cholerae colonization was decreased $\sim 4.47$-fold in the presence of transplanted $B$. vulgatus. Although the difference between these two groups was less pronounced than what was observed in experiments with adult mice, $V$. cholerae colonization and 


\begin{tabular}{|c|c|c|c|c|c|c|c|c|c|}
\hline Phylum & Species & Con & SM & VAN & $\mathrm{CL}$ ICon- $\mathrm{Vc}$ & $S M-V c$ & VAN-Vc & $\overline{C L-V c}$ & \multirow{2}{*}{$\begin{array}{l}\text { Relative abundance } \\
\text { in each group } \\
\begin{array}{|l}50 \% \\
40 \sim 50 \% \\
30 \sim 40 \%\end{array}\end{array}$} \\
\hline Bacteroidetes & $\begin{array}{c}\text { Bacteroides vulgatus } \\
\text { Parabacteroides goldsteinii } \\
\text { Bacteroides caccae }\end{array}$ & & $\begin{array}{l}\square \\
\square\end{array}$ & $\square$ & $\square$ & $\begin{array}{r}\square \\
\square\end{array}$ & $\square$ & $* *$ & \\
\hline Deferribacteres & Mucispirillum schaedleri & $\begin{array}{ll}\square \\
\end{array}$ & & $\square$ & $\square$ & & & & $10 \sim 20 \%$ \\
\hline Verrucomicrobia & Akkermansia muciniphila & & $\square$ & $\square$ & 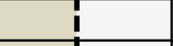 & \begin{tabular}{|l|} 
\\
\end{tabular} & $\square$ & & $5 \sim 10 \%$ \\
\hline Proteobacteria & $\begin{array}{l}\text { Proteus vulgaris } \\
\text { Escherichia coli } \\
\text { Vibrio cholerae }\end{array}$ & & & $\begin{array}{l}\square \\
\square \\
\end{array}$ & i & & \begin{tabular}{|l|l}
$\square$ \\
$\square$
\end{tabular} & $\begin{array}{ll} \\
\\
\end{array}$ & $\square \quad 1 \sim 5 \%$ \\
\hline \multirow{6}{*}{ Firmicutes } & $\begin{array}{l}\text { Vallitalea pronyensis } \\
\text { Clostridium saccharolyticum } \\
\text { Clostridium asparagiforme }\end{array}$ & $\square$ & $\square$ & & $\square$ & \begin{tabular}{|c} 
\\
\end{tabular} & & & \\
\hline & $\begin{array}{c}\text { Clostridium amazonense } \\
\text { Lactobacillus animalis } \\
\text { Clostridium thermosuccinogenes }\end{array}$ & & & & & & \begin{tabular}{|l} 
\\
\end{tabular} & & \\
\hline & $\begin{array}{c}\text { Christensenella massiliensis } \\
\text { Saccharofermentans acetigenes } \\
\text { Eubacterium siraeum }\end{array}$ & & & \begin{tabular}{|l}
$\square$ \\
$\square$
\end{tabular} & $\square$ & & \begin{tabular}{|l} 
\\
\end{tabular} & \begin{tabular}{|l} 
\\
\end{tabular} & \\
\hline & $\begin{array}{c}\text { Neglecta timonensis } \\
\text { Acutalibacter muris } \\
\text { Enterococcus saigonensis }\end{array}$ & & & & $\begin{array}{l}\square \\
\square \\
\end{array}$ & & & $\begin{array}{l}\square \\
\square \\
\\
\end{array}$ & \\
\hline & $\begin{array}{l}\text { Lachnoclostridium pacaense } \\
\text { Flintibacter butyricus } \\
\text { Oscillibacter ruminantium }\end{array}$ & $\square$ & $\square$ & & $\square$ & $\begin{array}{l}\square \\
\square \\
\square \\
\end{array}$ & & & \\
\hline & $\begin{array}{c}\text { Clostridium cocleatum } \\
\text { Anaerotruncus colihominis }\end{array}$ & & & & i & & $\square$ & $\square$ & \\
\hline $\begin{array}{l}\text { Fig. } 3 \text { Species-level } \\
\text { microbial species at } \\
\text { conditions. Con, SM } \\
\text { and CL-Vc indicate } \\
\text { (brown) and Proteo } \\
\text { a relative abundanc } \\
\text { color-coded boxes. } \\
\text { elimination of Bacte }\end{array}$ & $\begin{array}{l}\text { crobiota populations in response } \\
\text { phylum (far-left column) and } \\
\text { N, and } C L \text { in the first low indica } \\
\text { ples of the same groups followi } \\
\text { teria (gray) phyla and } 17 \text { species } \\
>\% \text { of the total microbiota po } \\
\text { example, brown boxes mean th } \\
\text { les vulgatus is indicated with **, }\end{array}$ & $\begin{array}{l}\text { es (s } \\
\text { nicro } \\
\text { l. cho } \\
\text { ongi } \\
\text { tion } \\
\text { he ah } \\
\text { le a }\end{array}$ & $\begin{array}{l}\text { sam } \\
\text { ae in } \\
\text { to th } \\
\text { re se } \\
\text { dan } \\
\text { box }\end{array}$ & $\begin{array}{l}\text { of } \\
\text { on. } \\
\text { rmic } \\
\text { ed ar } \\
\text { a gi } \\
\text { ootec }\end{array}$ & $\begin{array}{l}\text { d after } V \text {. choler } \\
\text { Is are presented } \\
\text { esponding antil } \\
\text { e species were } \\
\text { (orange) phylu } \\
\text { displayed. Relati } \\
\text { species under } \\
{ }^{*} \text { indicates } 8.8\end{array}$ & $\begin{array}{l}\text { ae infect } \\
\text { by colo } \\
\text { biotic tre } \\
\text { determi } \\
\text { um were } \\
\text { ve abun } \\
\text { a specif } \\
1 \% \text { V.ch }\end{array}$ & $\begin{array}{l}\text { ion. Distr } \\
\text { rrs in } 8 \text { di } \\
\text { atments } \\
\text { ned to b } \\
\text { identifie } \\
\text { dance of } \\
\text { ic conditi } \\
\text { olerae oc }\end{array}$ & $\begin{array}{l}\text { bution o } \\
\text { fferent ex } \\
\text { and Con- } \\
\text { elong to } \\
\text { d. Only b } \\
\text { each spe } \\
\text { on is }>5 \\
\text { cupancy }\end{array}$ & $\begin{array}{l}\text { f commensal } \\
\text { perimental } \\
\text { Vc, SM-Vc, VAN-Vc, } \\
\text { Bacteroidetes } \\
\text { acterial species with } \\
\text { cies is indicated by } \\
\% \text {. A complete }\end{array}$ \\
\hline
\end{tabular}

infection-induced fluid accumulation were both reduced significantly in B. vulgatus-transplanted infant mice (Fig. 4d).

\section{Metabolome profiles revealed the characteristic metabolites that directly modulate intestinal $V$. cholerae growth}

We next conducted a metabolome analysis to examine whether metabolomic profiles are altered in response to CL treatment and/or $V$. cholerae infection. We postulated that alterations of metabolite levels influenced host susceptibility to $V$. cholerae infection. Cecal contents were subject to sample preparation for CE-TOF/ MS, as described in the "Methods" section. Among the metabolites detected in our analysis, a total of 273 metabolites were successfully quantified and their relative quantities between samples are presented in a heat map (Fig. 5). A table that lists all 273 metabolites and their relative amounts in four samples is shown as supplementary information (a supplementary excel file). Of the many differences between the metabolomic profiles of each group, the most noticeable one is that red and green line clusters representing compounds in large and small quantities, respectively, are generally reversed in control SPF vs. CL-treated group (column 1 vs. 3, Fig. 5). Such striking alterations in metabolome profiles can be interpreted as a consequence of the dramatic microbiota compositional changes, as shown in Fig. 1. Interestingly, a smaller degree of profile change was observed in response to $V$. cholerae infection in both experimental groups (column 1 vs. 2 and column 3 vs. 4, Fig. 5). These results demonstrate that (i) CL treatment induces more substantial changes in metabolome profiles than does $V$. 

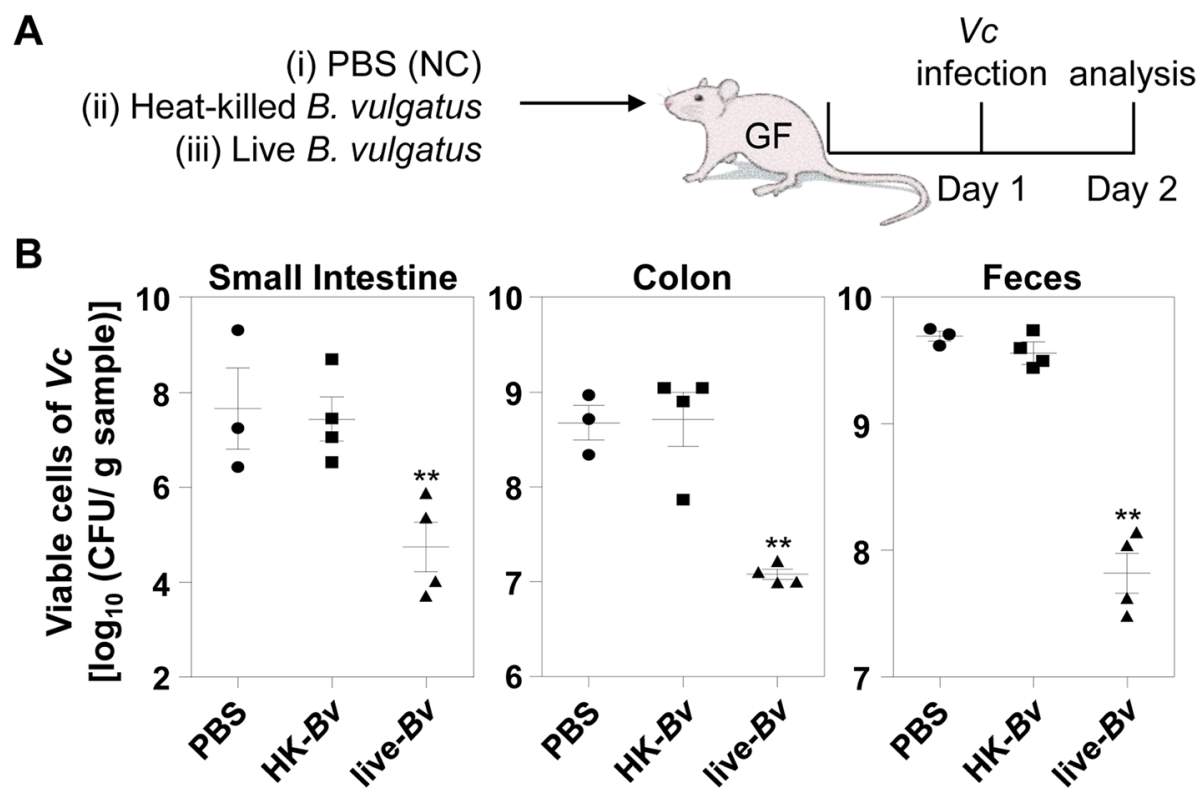

C

Vc

infection analysis

(ii) Live $B$. vulgatus

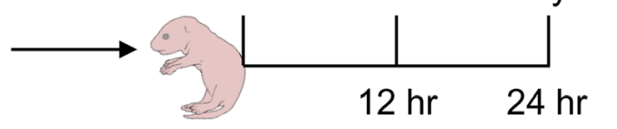

D
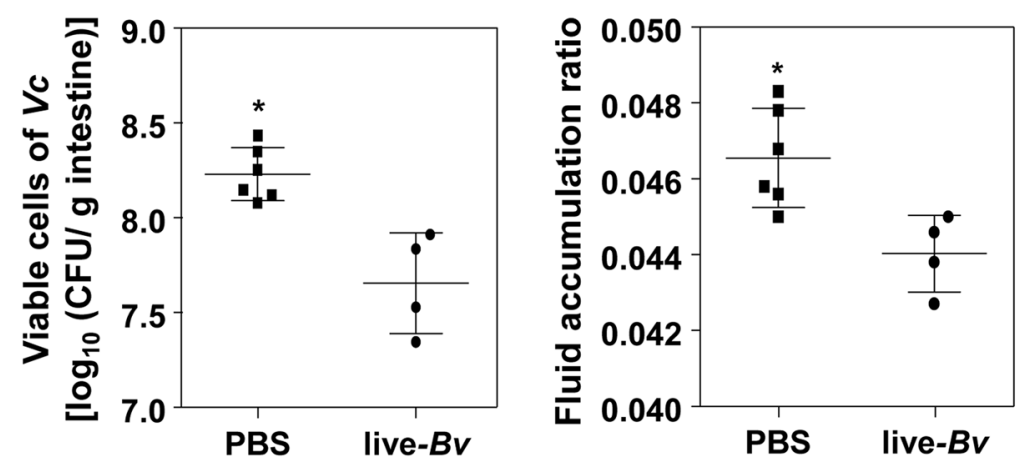

Fig. 4 B. vulgatus inhibits $V$. cholerae growth in vivo. a Schematic diagram of the experimental procedure. Germ-free mice (C57BL/6, 8 9 weeks old, $n=3$ or 4 per group) ingested $50 \mu \mathrm{LPBS}(n=3)$, heat-inactivated (autoclaved) B. vulgatus cell suspension $(n=4)$, or live $B$. vulgatus cell suspension $(n=4)$. The $B$. vulgatus cell suspensions contained $2 \times 10^{9} \mathrm{CFU} / \mathrm{mL}$. At $24 \mathrm{~h}$ post-association, mice were challenged with $V$. cholerae infection $\left(5 \times 10^{8} \mathrm{CFU}\right)$ for $24 \mathrm{~h}$. b Viable $V$. cholerae cells in $\mathrm{Sl}$, colon, or fecal pellets were enumerated and presented in log-scale. ${ }^{* *} P<0.001$ versus bacterial CFU in the control and heat-inactivated BV groups. c Schematic diagram of the experimental procedure. Five-day-old infant mice ( $n=4$ or 6 per group) ingested $50 \mu \mathrm{L}$ PBS $(n=6)$ or B. vulgatus cell suspension $(n=4)$. The $B$. vulgatus cell suspensions contained $1 \times 10^{9} \mathrm{CFU} /$ $\mathrm{mL}$. At $12 \mathrm{~h}$ post-association, mice were challenged with $\mathrm{V}$. cholerae infection $\left(1 \times 10^{8} \mathrm{CFU}\right)$ for $12 \mathrm{~h}$. $\mathbf{d}$ Viable $V$. cholerae cells in intestinal extract were enumerated and presented in log-scale. ${ }^{* *} P<0.05$ versus bacterial CFU in the control group. At the end of the infection period, fluid accumulation ratio was calculated by the equation of (intestine weight)/[(total body weight)-(intestine weight)]. ${ }^{*} P<0.05$ versus the control group

cholerae infection, and (ii) CL-induced changes on metabolite level might have created an environment that strongly facilitates $V$. cholerae growth and pathogenesis.

Table 1 shows a list of metabolites that were detected in the greatest quantities in control SPF (compounds 1 13) or CL-treated (compounds 14 27) mice. Out of the 13 molecules detected in the SPF mouse ceca, all except for cholic acid were not detected in CL-treated mice. It is of particular interest that (iso) butyric acid, propionic acid, and (iso) valeric acid, collectively termed short-chain fatty acid (SCFA) are among the most abundantly detected metabolites in control group (Table 1). Fourteen compounds (14 27), whose relative quantities are greater than 1.0E-03, 


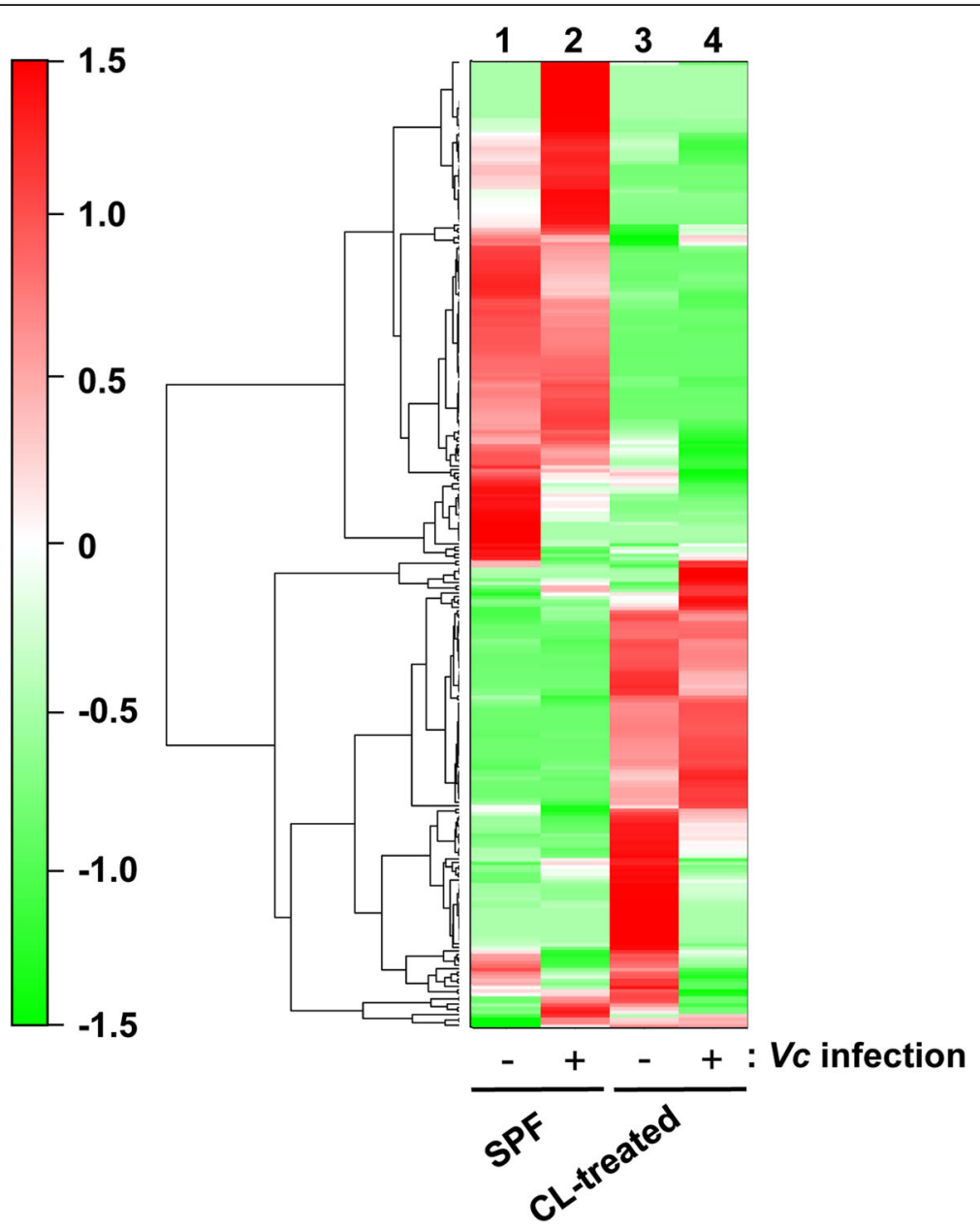

Fig. 5 Heat map constructed from hierarchical cluster analysis (HCA) of cecal metabolites. Metabolites $(n=273)$ that exhibit similar detection patterns in four samples are clustered, and the distance between clusters is shown as a tree diagram. The degree of red or green color indicates a larger or smaller amount of a given metabolite. Columns 1 and 2 display metabolome profiles of regular SPF mice, while 3 and 4 shows those of CL-treated groups. Columns 2 and 4 are metabolome profiles after $V$. cholerae infection in each group

were selected and presented as dominant metabolites in the CL-treated group (Table 1, bottom portion). Relative quantities of those molecules were either nondetectable (compounds 14, 21, 23, and 27) or considerably smaller in the control SPF group. These results further support the notion that distinct profile change occurred in response to CL treatment. We next examined how $V$. cholerae growth is controlled by each of these major compounds. Of the 27 listed compounds, 17 were selected based on the availability and we monitored $V$. cholerae in vitro growth in M9 media supplemented with each metabolite. Growth curve experiments shown in Fig. 6 were conducted to reveal compounds that can inhibit $V$. cholerae growth. M9 media used in this particular set of growth experiments contain $0.4 \%$ glucose as primary carbon source, thereby enabling us to monitor whether the extraneously added metabolite promotes or inhibits $V$. cholerae growth. Seven metabolites shown in panels $\mathrm{K} \sim \mathrm{Q}$ clearly inhibited $V$. cholerae growth. Importantly, these compounds were present in the largest quantities in regular SPF mouse intestines, but either absent or barely detectable in CLtreated mouse intestines (Table 1). Prominent growth promotion was observed when the culture medium included NAG (Fig. 6b), a metabolite detected at sufficiently high level in CL-treated mice. Supplementation with urea (Fig. 6a), glucaric acid (Fig. 6f), putrescine (Fig. 6i), or fumaric acid (Fig. 6j) resulted in a slight growth stimulation, especially during the early stage of growth. Again, these metabolites were detected more abundantly in CLtreated mice, than in the SPF control mice (Table 1). When N16961 growth was assessed in M9 media with an indicated metabolite as the sole carbon source, each metabolite's ability to stimulate $V$. cholerae growth was clearly demonstrated (Fig. 7). Consistent with result shown in Fig. 6b, NAG was most potent in stimulating $V$. 
Table 1 Metabolites found to be dominant in regular SPF and CL-treated mouse intestines and their impacts on V. cholerae growth

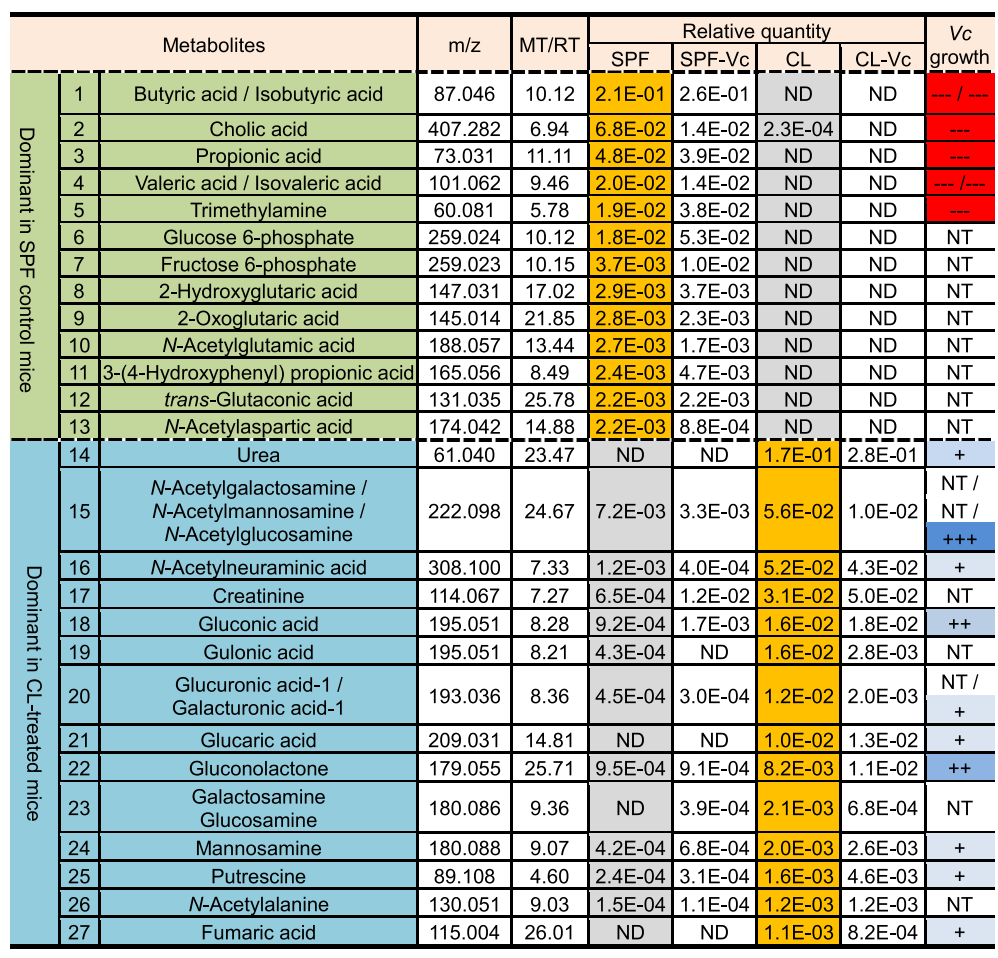

Twenty-seven metabolites that were present exclusively in either group were selected among 273 compounds. Metabolites detected most abundantly in the control group (No.1 13) or in the CL-treated group (No.14 27) are listed in the order of quantity. The growth-inhibiting or growth-promoting capability of a given metabolite is displayed semi-qualitatively with a minus $(-)$ or plus $(+)$ sign, respectively. For example, " + " indicates a metabolite with mild growth-promoting capability, whereas "---" indicates that the metabolite induced strong growth suppression. ND not detected, NT not tested, $m / z$ mass-to-charge ratio, MT migration time, $R T$ retention time, Vc Vibrio cholerae. Relative quantity of a metabolite is calculated by the equation: (peak area of the metabolite)/(peak areas of internal standards $\times$ sample amount). Peaks are assigned based on the signal-to-noise ratio $(S / N)$ of 3 or above.

cholerae growth, with $\mathrm{OD}_{600}$ values reaching $\sim 1.05$ (Fig. 7b). Next to NAG, gluconic acid (Fig. 7d) and gluconolactone (Fig. $7 \mathrm{~g}$ ) were effective at promoting $V$. cholerae growth. As expected, seven inhibitory metabolites (cholic acid, butyric acid, isobutyric acid, propionic acid, valeric acid, isovaleric acid, and trimethylamine) still prevented growth of $V$. cholerae (Fig. $7 \mathrm{k}-\mathrm{q}$ ). Together, these results illustrate that CL-induced changes in the host intestine are clearly reflected on metabolite level and such major alterations of metabolomic profiles significantly affect $V$. cholerae intestinal growth.

\section{CL-treated adult mice can serve as a model for investigating $V$. cholerae pathogenesis}

Our results demonstrate that infection-induced phenotypes, such as intestinal colonization and fluid accumulation, are clearly observed in CL-pretreated adult mice. Therefore, we finally examined whether or not CL-treated mice can be utilized as a model to delineate $V$. cholerae infection. To address this issue, two groups of mice were infected with 1:1 mixture of N16961 and its $\triangle t c p A$ mutant, a well-characterized $V$. cholerae mutant defective in colonizing the intestine [40]. We then compared colonization capabilities of these two strains by calculating the competitive index. In this particular set of experiments, fluid accumulation and bacterial colonization were again substantially increased in CLtreated mice, but not in PBS-treated control group (Additional file 3: Figure S3). $V$. cholerae cells were completely eliminated in three out of six mice in control group (red arrow lines, Fig. 8a). Importantly, $\triangle t c p A$ mutant exhibited significantly reduced colonization capabilities, in comparison to WT N16961 (Fig. 8b). The average values of the competitive indices $(\Delta t c p A / \mathrm{WT})$ were $\sim 0.29$ and $\sim 0.28$ in the small intestine and cecum of CL-treated mice, respectively. In contrast, meaningful differences in colonization capabilities between two strains were not detected, when untreated adult mice were used (Fig. 8b). These results suggest that CL pretreatment not only induces active bacterial colonization but also creates a valuable environment we can exploit to better understand the pathogenesis of $V$. cholerae.

\section{Discussion}

A long-standing unresolved question in cholera research is how $V$. cholerae has evolved as a human-specific pathogen in nature. It has been of particular 


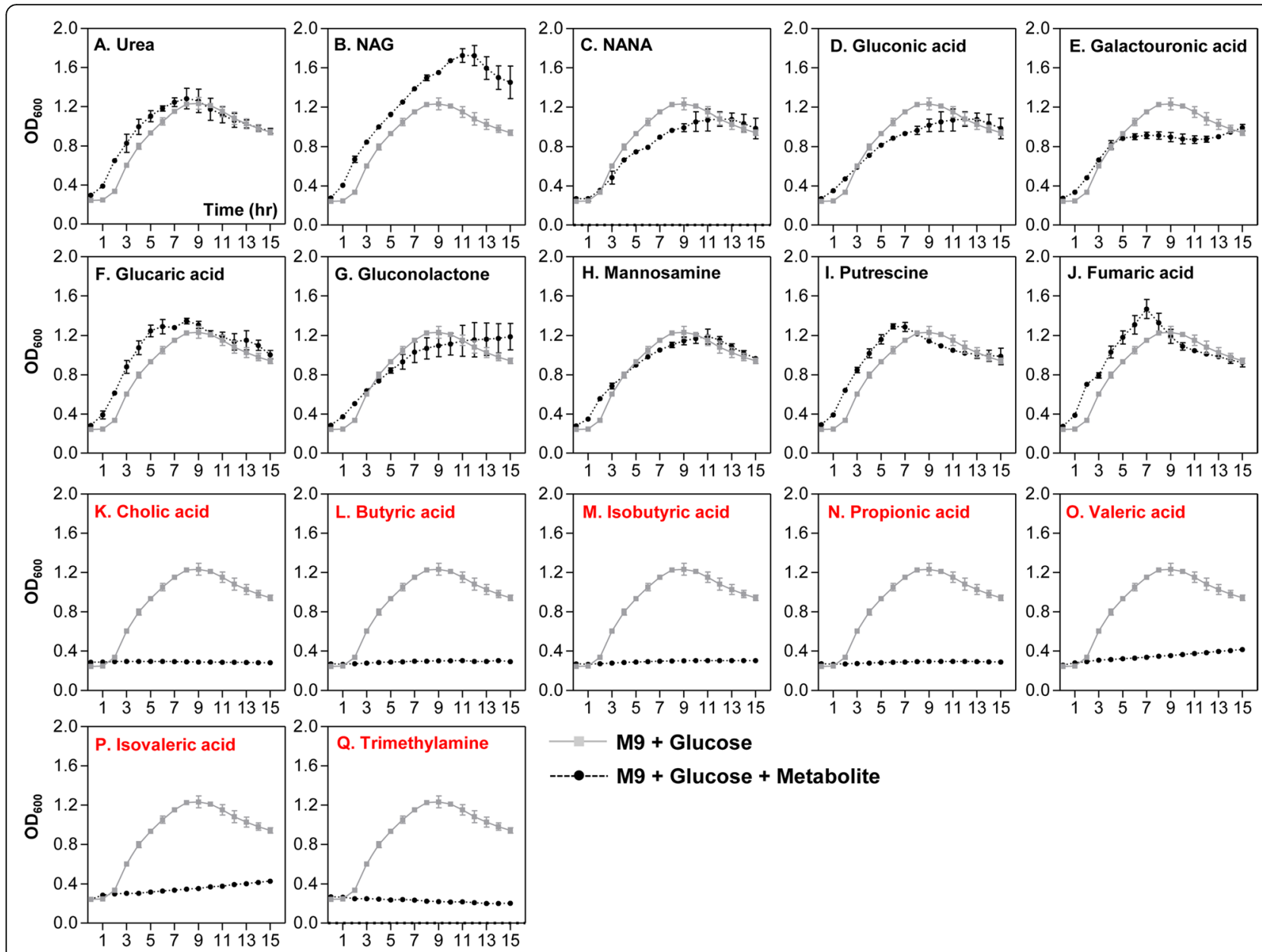

Fig. 6 Effects of selected metabolites on the inhibition of $V$. cholerae growth in vitro. N16961, a 7th pandemic $V$. cholerae strain, was grown in the presence of indicated metabolites in $\mathrm{M} 9$ media. A total of 17 metabolites were tested. Medium pH was neutralized by adding $\mathrm{NaOH}$ or $\mathrm{HCl}$, when necessary. $\mathrm{OD}_{600}$ values were measured to monitor bacterial growth every $2 \mathrm{~h}$. M9 media contains $0.4 \%$ glucose as a primary carbon source. Lines in gray show bacterial growth in M9+glucose (M9G), while black lines indicate bacterial growth in M9G supplemented with indicated metabolites. Cholic acid (panel k), Butyric acid (panel I), Isobutyric acid (panel $\mathbf{m}$ ), Propionic acid (panel $\mathbf{n}$ ), Valeric acid (panel o), Isovaleric acid (panel $\mathbf{p}$ ), and Trimethylamine (panel $\mathbf{q}$ ) are highly capable of inhibiting N16961 growth

interest that GM1 ganglioside, a lipid molecule that binds to CT, is present not only on human intestinal epithelium but also mouse intestinal epithelium [41, 42]. This notion suggests that CT, the most important virulence determinant in $V$. cholerae, should also be active in mouse intestine. However, adult mice are naturally resistant to $V$. cholerae infection. We hypothesized that understanding compositional differences between human and mouse intestinal microbiotas would help address this important issue of host tropism.

Our 16S rRNA gene sequencing data (Figs. 1 and 3) show that approximately $80 \%$ of the C57BL/6 mouse gut microbiota belongs to the Bacteroidetes phylum. Virtually complete elimination of the phylum induced by $\mathrm{CL}$ treatment is essential for infecting the murine host with $V$. cholerae. However, for our findings to be clinically relevant, it needs to be first established that the mouse gut microbiome is, to a sufficient degree, reflective of the human gut microbiome. Previous studies have reported predominantly high relative abundances of the Bacteroidetes phylum in both mouse and human gut microbiomes [43-46]. Furthermore, there is evidence that $B$. vulgatus isolates from human and other animal hosts are indistinguishable based on their 16S rRNA gene sequences [47], suggesting phylogenetic proximity between mouse- and human-gut resident $B$. vulgatus strains. Nonetheless, the composition of a gut microbiome does not necessarily reflect its functional capacity [46]. Additionally, even though the mouse gut microbiome closely resembles that of humans at the phylum level, the similarity dissipates on lower taxonomic levels, as families and genera that comprise the predominant Bacteroidetes phylum are quite different between mice and humans [44]. To 


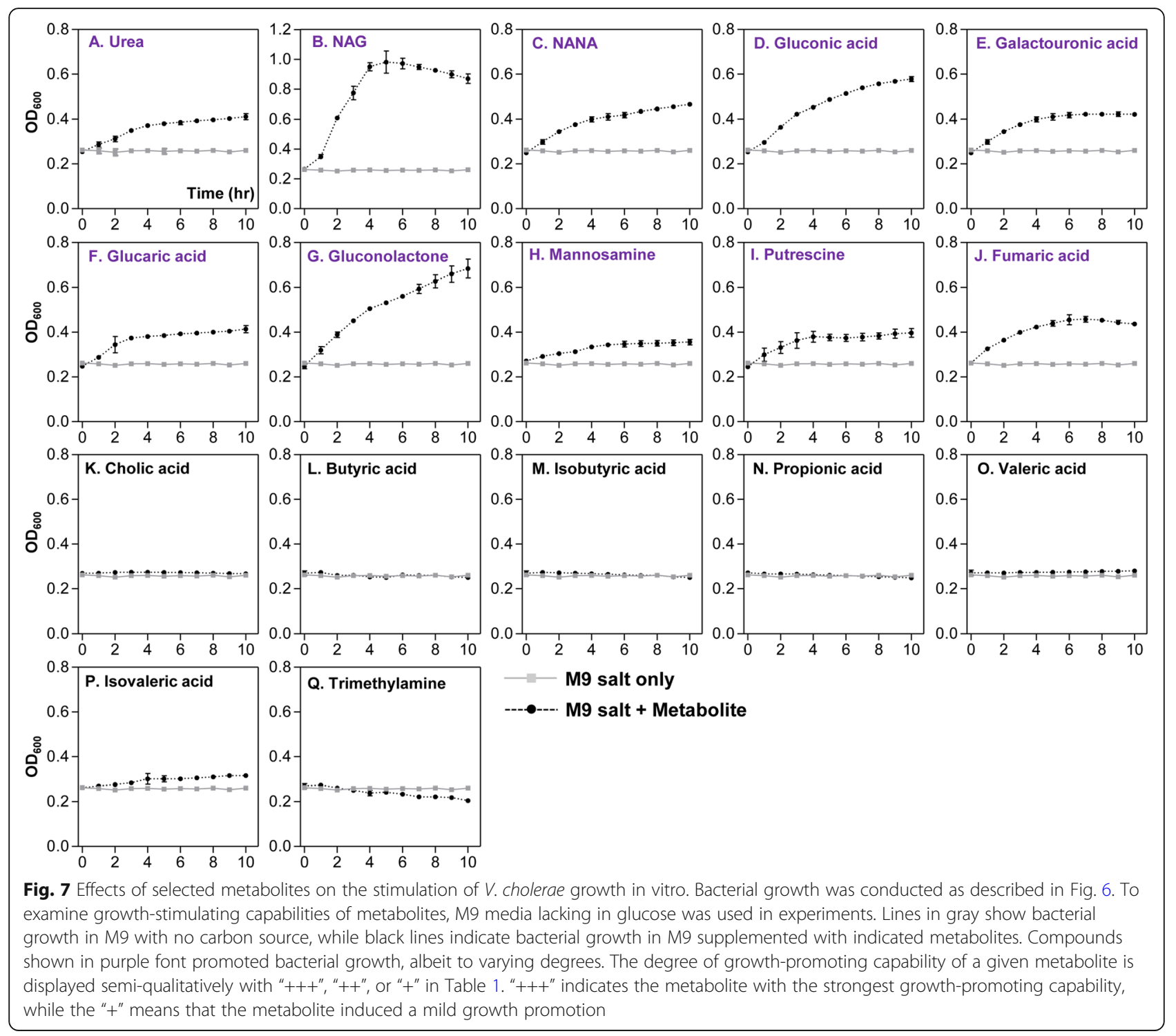

compensate for such limitations, we have demonstrated that our key findings are reproduced when GF mice are mono-associated with $B$. vulgatus and infected with $V$. cholerae (Fig. 4a, b). Given such considerations, it is likely that the relative abundance of $B$. vulgatus is an important determining factor for $V$. cholerae infectivity not only in murine hosts but in humans as well. An important question would be how abundantly $B$. vulgatus cells that share similar functions with mouse isolates are present in human intestine.

Based on our species-level population changes, Escherichia coli explosively proliferated during CL treatment and became the most dominant component (Fig. 3). This finding suggests that elevated susceptibility to $V$. cholerae infection in CL-pretreated mouse could also be due to the presence of $E$. coli cells in large quantity. In line with this notion, an E. coli strain with robust catalase activity was found to stimulate enhanced $V$. cholerae intestinal colonization in neonatal mice [1]. In the same work, increased $V$. cholerae colonization occurred in VAN-treated adult mice [1]. In the current study, however, cholera-like symptoms including fluid accumulation in the small intestine and production of watery fecal matter were not observed in the VAN-treated group (Fig. 1k). We therefore conclude that the loss of B. vulgatus is a much more critical determinant over increased $E$. coli for host susceptibility to $V$. cholerae infection.

In the present study, we found that $\mathrm{CL}$ treatment increases $V$. cholerae infectivity in the normally resistant adult mice. Previous studies have reported that antibiotic administration triggers dysbiosis in the gut microbiota [48, 49], and such perturbations lead to suppression of host resistance against various enteropathogenic infections. Since CL treatment 


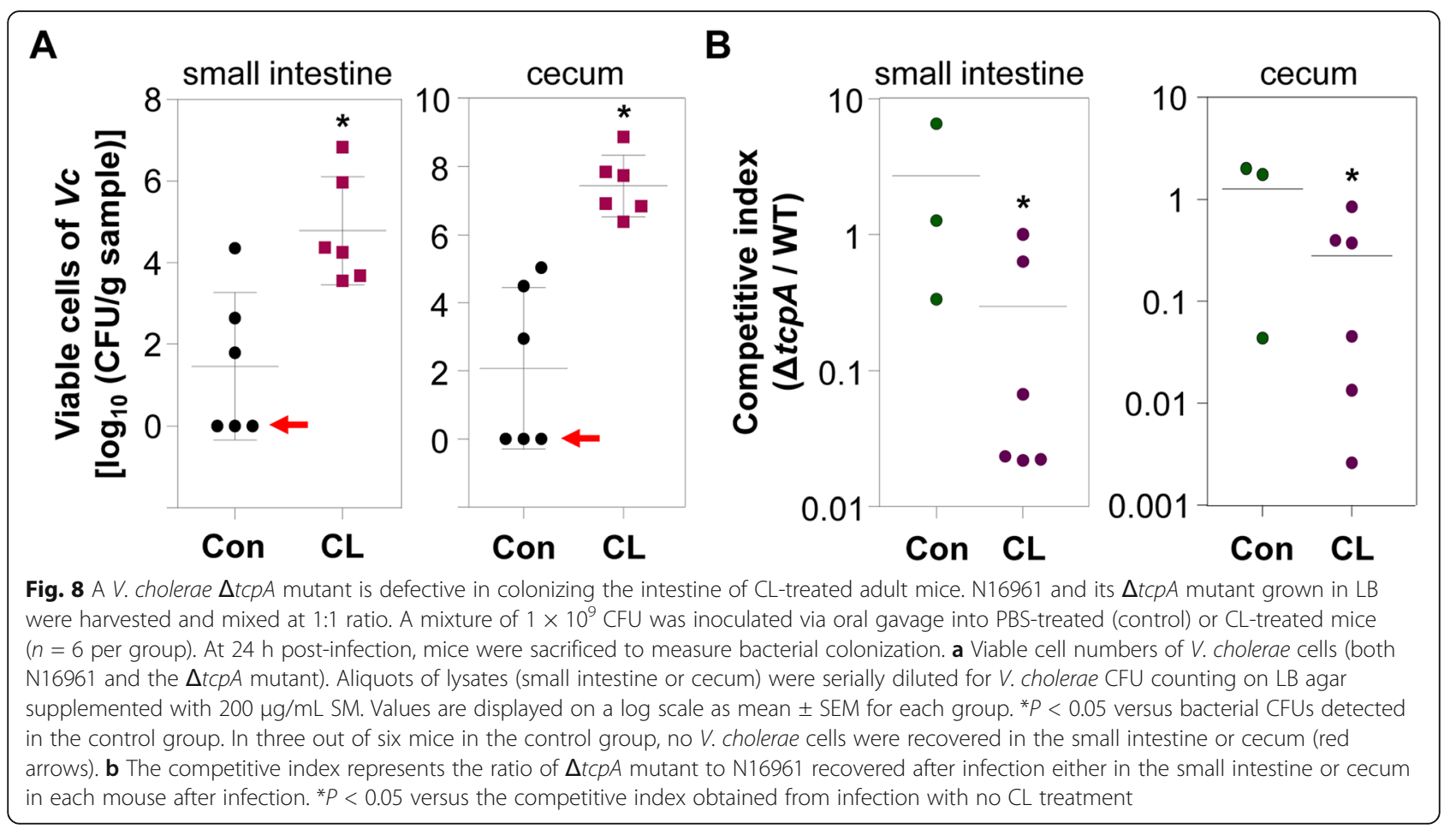

results in significant perturbation of the gut microbiome, it is conceivable that this model would demonstrate increased susceptibility to infections by other enteric pathogens such as Salmonella enterica serovar Typhimurium, Citrobacter rodentium, and Clostridium difficile. However, for the aforementioned enteric pathogens, there already exists a wellestablished adult mouse model $[50,51]$. Here, we suggest a simple and convenient animal model to study $V$. cholerae infection. The adult mouse model treated with oral CL consistently exhibited cholera-like symptoms. Out of the several already existing animal models for cholera research, the suckling mouse model is considered to be a descriptive model that manifests the cholera-characteristic watery diarrhea. However, the model is not adequate for use in vaccine research, because suckling mice have yet to develop adaptive immunity [52]. Moreover, the susceptibility of suckling mice to $V$. cholerae infection might be an artifact caused by their immature immune system, although this idea has not yet been fully verified. Therefore, an infection model using adult mouse has been required for the purpose of unveiling the pathophysiological mechanisms that $V$. cholerae employs to induce cholera in the host intestine [53]. However, intestinal fluid secretion and continuous colonization of $V$. cholerae do not readily occur in adult mice harboring intact gut microflora $[54,55]$. For this reason, the ligated ileal loop model that involves surgical manipulation of the animal's intestine has been proposed as an alternative [56]. Our CL-induced adult mouse model, which can be established by a simple treatment, clearly reproduces typical cholera symptoms observed in humans, such as the severe watery diarrhea, extensive fluid accumulation, and $V$. cholerae colonization within the intestinal mucus layer. Furthermore, in our model, CT has been detected in the intestinal fluid, at a level sufficient to elicit intestinal fluid secretion (Fig. 2c). In addition, the competitive index of $\triangle t c p A / W T$ showed that TCP, a verified colonization factor of $V$. cholerae in human intestine $[57,58]$, is also essential for the induction of illness in adult mice (Fig. 8). To date, the importance of TCP has been explored only in infant rabbits [53] and infant mice [59].

Host-derived mucus glycan is a nutrient source utilized exclusively by the gut microbiota. Considering the microbial competition for nutritional resources in the intestinal tract and constant release of glycan into the lumen by epithelial cell turnover [60-62], the ability of select microbes to nutritionally exploit the glycans confers a conceivable advantage toward their survival and proliferation. A large proportion of glycan structures consist of $\mathrm{N}$-acetyl amino sugars, such as $\mathrm{N}$-acetylgalactosamine (GalNAc), $N$-acetylglucosamine (GlcNAc, NAG), and $N$-acetylneuraminic acid (Neu5Ac, NANA). Monosaccharides are produced as the result of mucin glycans being catabolized by the gut microbial species equipped with glycosidase genes. Bacteroides has evolved to express glycosidase genes which allow them to perform mucin degradation to full completion, and thus become well-adapted to thrive in the intestine [61-63]. The anaerobes that belong to the Bacteroidetes phylum and Clostridia produce pyruvate through glycolysis [61, 64]. Under the anoxic conditions of the intestine, the gut microbiota obtains additional energy 
by exploiting the pyruvate through anaerobic catabolism. Predominant end products of the anaerobic fermentation are short-chain fatty acids (SCFAs), such as propionic acid, butyric acid, and valeric acid [61].

In this study, the dramatic changes in the cecal metabolomes of CL-treated mice strongly correlate with the compositional shift of the microbiota. Depletion of SCFAs and relatively high occurrence of amino sugars in the ceca of CL-treated mice indicate that catabolism of mucin glycan, a proliferation strategy for microbes belonging to the Bacteroidetes phylum, became inactive upon CL treatment.

Utilization of the host glycan is also important for the invasion of $V$. cholerae into the host intestine. When the pathogen enters the intestinal tract, the next strategy for its proliferation is to colonize the epithelial cells covered with a viscous layer of mucus. $V$. cholerae effectively responds to the mucus glycan and catabolize the polysaccharides to acquire competitive advantage in host intestine [65-68]. Glucosamine-6-phosphate (GlcNP-6), a common intermediate molecule shared between the catabolic pathways of GlcNAc and Neu5Ac, was reported to be indispensable for $V$. cholerae motility [68]. Chemotaxis mediated by motility toward the nutrient sources is important for colonization and proliferation of $V$. cholerae [68].
Consistent with this notion, depletion of sialic acid (Neu5Ac) transporters (encoded in VC1777-1779) attenuates colonization [69]. During this process, $V$. cholerae needs to overcome a number of challenges such as acid stress, antimicrobial peptides, host immune response, and SCFAs [64, 66, 68]. The reduction of intestinal SCFAs in patients with diarrhea and recovery of normal SCFA levels after conventional treatment support that the SCFAs could be a contributing factor to eubiosis of the intestinal microbiota [70]. In general, SCFAs are considered to be an effective barrier against pathogenic invasion, although some enteric pathogens exploit SCFAs to sense their surroundings and to decide whether or not to express their arsenal of virulence factors [71].

Our results suggest that catabolism of $\mathrm{N}$-acetyl amino sugars and subsequent accumulation of SCFAs in the distal intestine are largely managed by specific gut microbiota (Fig. 3). Elimination of the anaerobic Bacteroides and Clostridium genera caused by CL treatment results in drastic changes of intestinal metabolites as summarized in Fig. 9. Especially, propionic and butyric acid mainly produced by Bacteroides and Clostridium, respectively, are dramatically reduced after CL treatment, thus leaving a variety of metabolites (NAG, NANA, gluconate, gluconolactone, etc.)

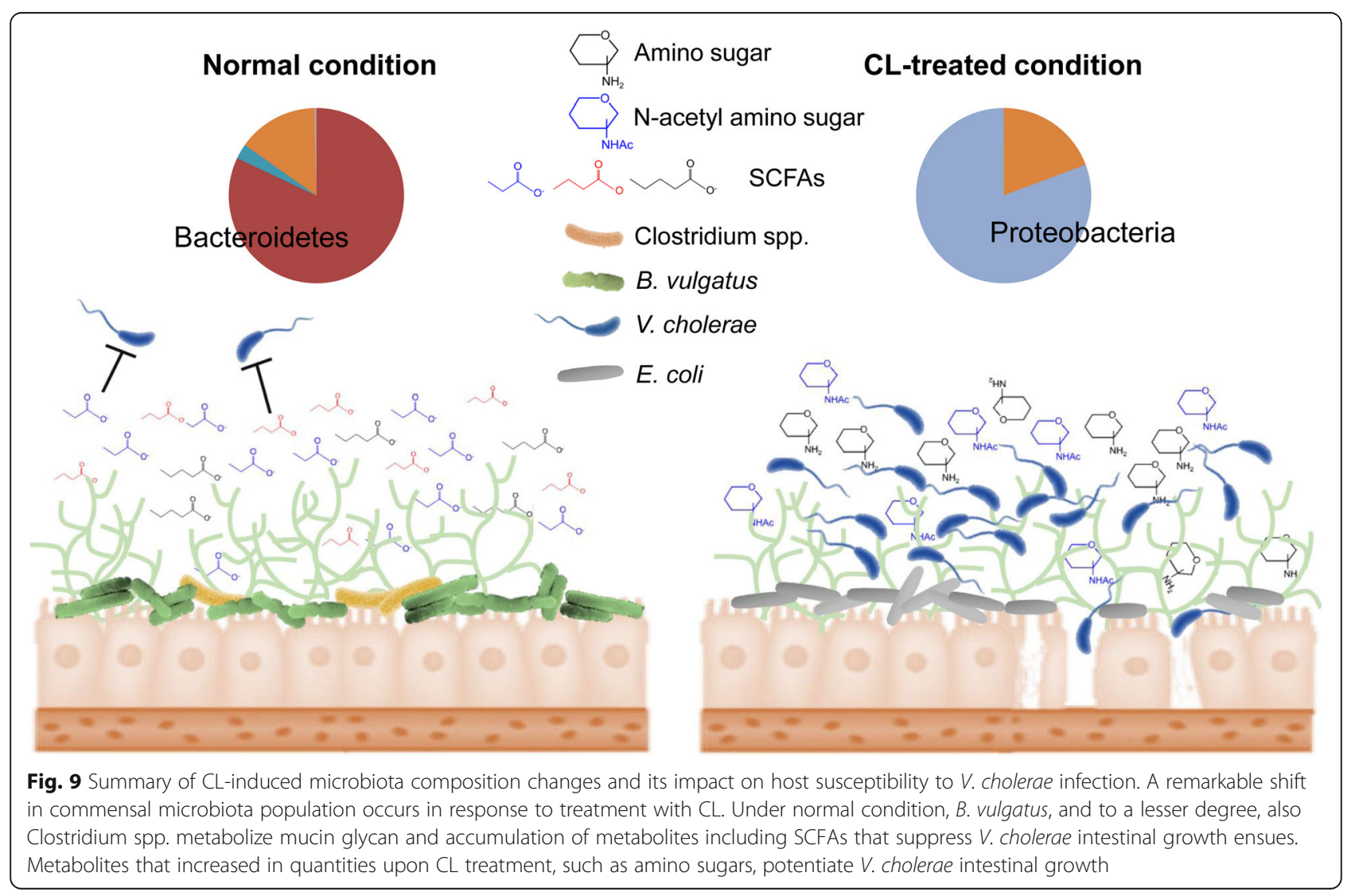


available for utilization by $V$. cholerae (Fig. 9). Of particular note, gluconate and its intermediate metabolites were found to enhance the colonization and virulence of $V$. cholerae within the intestine [72]. In the perturbed intestinal environment, $V$. cholerae readily acquires nutrient sources as there is no competition with the host glycan-degrading and thus SCFA-producing gut microbiota (mainly Bacteroides and Clostridium) genera.

\section{Conclusions}

In conclusion, we identified a commensal bacterial species, B. vulgatus, that plays a significant role in inhibiting $V$. cholerae colonization in the adult mouse intestine. Moreover, we propose a simple antibiotictreated mouse model to delineate $V$. cholerae infection, which might prove a useful tool for cholera vaccine research. We hope that the results presented in the present study will stimulate future investigations to better understand the in vivo probiotic function of $B$. vulgatus against $V$. cholerae infection and to devise better strategies for tackling this virulent and clinically important human pathogen.

\section{Methods}

\section{Bacterial strains and growth condition}

Vibrio cholerae O1 serotype N16961 was used as a model pathogen in all experiments [73]. N16961 was routinely grown in Luria-Bertani (LB) broth $(10 \mathrm{~g}$ $\mathrm{NaCl}, 10 \mathrm{~g}$ tryptone, and $5 \mathrm{~g}$ yeast extract per $\mathrm{L}$ ) or on LB agar plates (15 g agar per L) at $37^{\circ} \mathrm{C}$ under aerobic condition. In order to selectively isolate N16961 from feces, SI, cecum, and colon, we used streptomycin (Duchefa) at a $200 \mu \mathrm{g} / \mathrm{mL}$ concentration. Another selective medium used to isolate $V$. cholerae from intestinal contents was thiosulfate-citrate-bile salt-sucrose (TCBS) medium. For enumeration of intestinal $V$. cholerae loads, mouse intestines were manually extracted from infected animals. The SIs, ceca, and colons were then homogenized in PBS (Sigma-Aldrich) and centrifuged at $1000 \mathrm{rpm}$ for $1 \mathrm{~min}$. The supernatants were serially diluted in PBS, and spotted on an LB agar plate containing streptomycin. B. vulgatus strains were isolated from C57BL/6 mouse intestines using Bacteroides Bile Esculin (KisanBio, Korea) agar plates. Subsequently, B. vulgatus strains were cultivated on Gifu Anaerobic Media (GAM) broth (KisanBio, Korea) in an anaerobic chamber maintained at $90 \% \mathrm{~N}_{2}, 5 \% \mathrm{CO}_{2}$, and $5 \% \mathrm{H}_{2}$. All anaerobic culture media were deoxygenated for at least $24 \mathrm{~h}$ prior to usage. RAPD analysis was performed using six different $B$. vulgatus colonies recovered independently from two different
CL-treated mice, following procedures described elsewhere [1].

\section{Mouse husbandry and antibiotic treatment}

Experiments were performed using both SPF and GF mice in a C57BL/6 genetic background. SPF mice were obtained from Orient Bio (Sungnam, Korea), and GF mice were generated by the Yonsei University College of Medicine GF mouse facility. Mice were provided sterile water and autoclaved food. GF status was confirmed by negative microbial growth in mouse feces. GF mice were bred and housed in flexible-film isolators (Class Biologically Clean Ltd., Madison, WI). When necessary, GF mice were transferred to isocages (Tecniplast Inc., Italy) for experimental grouping. All mouse experiments were conducted according to the guidelines provided by the Department of Animal Resources of Yonsei Biomedical Research Institute. The Committee on the Ethics of Animal Experiments at Yonsei University College of Medicine approved this study (Permit numbers, 20170210 and 2018-0250).

For the antibiotic treatment presented in Fig. 1, 8- to 9-week-old C57BL/6 female mice were administered either SM $(1 \mathrm{mg})$, VAN $(250 \mu \mathrm{g})$, and CL $(1 \mathrm{mg})$, or PBS by oral gavage once a day for 5 days. The dosage of the antibiotics was chosen to induce dysbiosis but not complete elimination of commensal species of the gut microbiota. Each oral gavage treatment did not exceed $100 \mu \mathrm{l}$. For the antibiotic treatment shown in Additional file 2: Figure S2, mice were treated with a single dose of CL (10 mg).

\section{V. cholerae infection and GF mouse manipulation}

$V$. cholerae cells grown aerobically overnight at $37{ }^{\circ} \mathrm{C}$ were diluted 100 -fold to inoculate fresh LB broth medium. The sub-cultured broth was incubated in a shaking incubator at $37{ }^{\circ} \mathrm{C}$ for $4 \mathrm{~h}$. Finally, $V$. cholerae cells were centrifuged at $13,000 \mathrm{rpm}$ for $10 \mathrm{~min}$, and the resulting pellet was suspended in sterile PBS to the concentration of $10^{10} \mathrm{CFU}$ per $\mathrm{mL}$. For infection, 8- to 9-week-old mice were orally infected with $50 \mu \mathrm{l}$ of $V$. cholerae cell suspension containing approximately $5 \times$ $10^{8} \mathrm{CFU}$ of $V$. cholerae cells. The fluid accumulation ratio was calculated by using the equation (intestine weight) / (total body weight-intestine weight). CT level was quantified by CT ELISA [38]. To enumerate $V$. cholerae cells in mouse feces, fecal pellets were physically disrupted to homogeneity, and fecal suspensions were serially diluted for CFU counting. Shortly after euthanasia, SI, cecum, and colon samples were manually extracted from each mouse and homogenized in 5 $\mathrm{mL}$ of PBS. The sample homogenates were centrifuged 
at low speed for remnant tissue removal, and the resulting supernatant was used for CFU counting.

FMT was performed to transplant the antibiotictreated gut microbiota into GF mice. SPF mice were treated either with CL or PBS as per the procedure described above, and fresh feces were collected $24 \mathrm{~h}$ post-antibiotic treatment. In each antibiotic-treated and control group, there were three mice, and two fecal pellets were collected from each mouse. The fecal pellets collected were pooled per group and suspended in $600 \mu \mathrm{l}$ of PBS. The suspension was quick-centrifuged to remove the debris, and the resulting supernatants were orally administered. Each GF mouse received $50 \mu \mathrm{l}$ of FMT solution by oral gavage. For $B$. vulgatus mono-association, $B$. vulgatus was first seeded with thawed stock and passaged at least twice and less than 4 times for maximum viability. B. vulgatus cells were grown in GAM broth under anaerobic conditions. The cultured $B$. vulgatus cells were resuspended in PBS, and $10^{8}$ CFU cells were delivered orogastrically to GF mice. Infant mouse infection was performed following procedures described previously [39].

\section{Competitive index assay}

To proceed with the competitive index assay, lacZnegative wild-type strain N16961 and lacZ-positive $\triangle t c p A$ were cultured as per the aforementioned $V$. cholerae culture protocol. Sub-cultured wild-type and $\triangle t c p A$ cells were centrifuged at 13,000 rpm for $2 \mathrm{~min}$ and then resuspended in PBS. Each strain was mixed at a 1:1 ratio, and $100 \mu \mathrm{l}$ of the prepared mixture (total $10^{9} \mathrm{CFU}$ ) was administered to 8- to 9-week-old female C57BL/6 mice (either untreated or CL-treated) by oral gavage. Each mouse was infected with $5 \times 10^{8} \mathrm{CFU}$ of each strain.

\section{Amplicon sequencing for microbiome profiling}

The extraction method for bacterial DNA was performed using a PowerMax Soil DNA Isolation Kit (MO $\mathrm{BIO})$. Each sequenced sample was prepared according to the Illumina 16S Metagenomic Sequencing Library protocols to amplify the V3 and V4 regions (519F806R). The DNA quality was measured by PicoGreen and Nanodrop. Input gDNA (10 ng) was PCR amplified. The barcoded fusion primer sequences used for amplifications were as follows: 341F: 5 '-CCTACG GGNGGCWGCAG, and 806R: 5'-GACTACHVGGGTATCTAATCC. The final purified product was then quantified using qPCR according to the qPCR Quantification Protocol Guide (KAPA Library Quantification kits for Illumina Sequencing platforms) and qualified using a LabChip GX HT DNA High Sensitivity Kit (PerkinElmer, MA, USA). Paired-end $(2 \times 300$ bp) sequencing was performed by Macrogen using the MiSeq $^{\text {Tix }}$ platform (Illumina, San Diego, CA, USA). For species identification, sequenced reads were aligned using blast and NCBI $16 \mathrm{~S}$ microbial database (version downloaded 2018.01.14). Taxonomy information was assigned only to OTUs that met both query coverage $>85 \%$ and identity percent $>85 \%$. OTUs that did not meet these two criteria were left unassigned. In general, microbial species was identified with 99\% identity with a reference sequence.

\section{Metabolite extraction}

For extracting ionic metabolites, approximately $50 \mathrm{mg}$ of cecal contents was dissolved in MilliQ water containing internal standards (H3304-1002, Human Metabolome Technologies (HMT), Tsuruoka, Yamagata, Japan) at a ratio of 1:9 (w/v). After centrifugation, the supernatant was centrifugally filtered through a Millipore 5000-Da cutoff filter (UltrafreeMC-PLHCC, HMT) to remove macromolecules $\left(9100 \times g, 4{ }^{\circ} \mathrm{C}, 60 \mathrm{~min}\right)$ for subsequent analysis with capillary electrophoresis time-of-flight mass spectrometry (CE-TOFMS).

\section{Metabolome analysis}

Metabolome analysis was conducted at HMT using the Basic Scan package with CE-TOFMS for ionic metabolites, based on the methods described previously $[74,75]$. Briefly, CE-TOFMS analysis was carried out using an Agilent $\mathrm{CE}$ capillary electrophoresis system equipped with an Agilent 6210 time-of-flight mass spectrometer, Agilent 1100 isocratic HPLC pump, Agilent G1603A CEMS adapter kit, and Agilent G1607A CE-ESI-MS sprayer kit (Agilent Technologies, Waldbronn, Germany). The systems were controlled by Agilent G2201AA ChemStation software version B.03.01 for CE (Agilent Technologies) and connected by a fused silica capillary (50 $\mu \mathrm{m}$ i.d. $\times 80 \mathrm{~cm}$ total length) with commercial electrophoresis buffer (H3301-1001 and H3302-1021 for cation and anion analyses, respectively, HMT) as the electrolyte. The spectrometer was scanned from $\mathrm{m} / \mathrm{z}$ 50 to 1000 [74]. Peaks were extracted using the automatic integration software MasterHands (Keio University, Tsuruoka, Yamagata, Japan) to obtain peak information including $\mathrm{m} / z$, peak area, and migration time (MT) for CE-TOFMS analysis [76]. Signal peaks corresponding to isotopomers, adduct ions, and other product ions of known metabolites were excluded, and remaining peaks were annotated according to the HMT metabolite database based on $m / z$ values with the MTs determined by TOFMS. Areas of the annotated peaks were then normalized based on internal standard levels and sample amounts to obtain relative levels of each metabolite. Hierarchical cluster analysis 
(HCA) was performed using the proprietary software supplied by HMT, PeakStat.

\section{$V$. cholerae in vitro growth curve experiments}

N16961 was grown in M9 media supplemented with 17 metabolites at $20 \mathrm{mM}$ each, with the exception of $\mathrm{N}$ acetylglucosamine (GluNAc), $\mathrm{N}$-acetylneuraminic acid (NANA), glucaric acid, gluconolactone, mannosamine, and cholic acid. These metabolites were added at concentrations of $4,1,4,10,1$, and $0.2 \mathrm{mM}$, respectively. N16961 growth was monitored by measuring $\mathrm{OD}_{600}$ values spectrophotometrically. To examine growth-inhibiting capabilities of the metabolites, N16961 was grown in M9 plus $0.4 \%$ glucose. However, M9 salt without glucose was used to assess the capabilities of the metabolites to promote the growth of $V$. cholerae.

\section{Statistical analysis}

Data are expressed as mean \pm standard error of the mean (SEM). Unpaired Student's $t$ test was used to determine whether differences between groups were significant. A $p$ value $<0.05$ was considered to indicate statistical significance. All experiments were repeated for reproducibility.

\section{Supplementary information}

Supplementary information accompanies this paper at https://doi.org/10. 1186/s40168-019-0746-y

Additional file 1: Figure S1. Effects of a single-dose treatment of $\mathrm{CL}$ on host resistance to $V$. cholerae infection.

Additional file 2: Figure S2. Random Amplified Polymorphic DNA (RAPD) analysis of $B$. vulgatus clones.

Additional file 3: Figure S3. A $V$. cholerae $\triangle t c p A$ mutant is defective in colonizing the intestine of $\mathrm{CL}$-treated adult mouse.

\section{Abbreviations}

CL: Clindamycin; CT: Cholera toxin; FA: Fluid accumulation; FMT: Fecal microbiota transplantation; GalNAc: N-acetylgalactosamine; GAM: Gifu anaerobic media; GF: Germ-free; HCA: Hierarchical cluster analysis; LB: LuriaBertani; NAG, GlcNAc: N-acetyl glucosamine; NANA: N-acetylneuraminic acid; SCFA: Short-chain fatty acids; SM: Streptomycin; SPF: Specific pathogen free; TCBS: Thiosulfate-citrate-bile salt-sucrose; TCP: Toxin-coregulated pilus; VAN: Vancomycin

\section{Acknowledgements}

Not applicable.

\section{Authors' contributions}

MYY and SSY conceptualized and designed the experiments. JSY, JHY, and GHK performed experiments and analyzed experimental results. JSY, JHY, GHK, MYY, and SSY drafted the manuscript. SM, KTN, and JHR provided germ-free mice and valuable comments. All authors read and approved the final manuscript.

\section{Funding}

This work was supported by grants from the National Research Foundation (NRF) of Korea, which is funded by the Korean Government, 2017R1A2A2A05019987 and 2017M3A9F3041233, 2019R1A6A1A03032869, and 2017R1A1A1A05001200. This work was also supported by Health Fellowship Foundation, as well as Korea Institute of Planning and Evaluation for Technology in Food, Agriculture, Forestry and Fisheries (IPET) through Agricultural Microbiome R\&D Program, funded by Ministry of Agriculture, Food and Rural Affairs (MAFRA) (918003041SB010).

\section{Availability of data and materials}

Unprocessed amplicon sequencing results (shown in Fig. 1 and Additional file 1: Figure S1) are provided in the supplementary spreadsheet. In addition, a complete list of metabolites and their relative quantities are displayed in the supplementary spreadsheet. Additional data will become available upon request.

\section{Ethics approval and consent to participate}

All animal studies were performed in compliance with the guidelines provided by the Department of Animal Resources of Yonsei Biomedical Research Institute. The Committee on the Ethics of Animal Experiments at the Yonsei University College of Medicine approved this study (permit number 2017-0210)

\section{Consent to publication}

Not applicable.

\section{Competing interests}

The authors declare that they have no competing interests.

\section{Author details}

${ }^{1}$ Department of Microbiology and Immunology, Yonsei University College of Medicine, 50-1 Yonsei-ro, Seodaemun-gu Seoul, Seoul 03722, Korea. ${ }^{2}$ Brain Korea 21 PLUS Project for Medical Sciences, Yonsei University College of Medicine, Seoul 03722, Korea. ${ }^{3}$ Severance Biomedical Science Institute, Yonsei University College of Medicine, Seoul 03722, Korea. ${ }^{4}$ Institute for Immunology and Immunological Diseases, Yonsei University College of Medicine, Seoul 03722, Korea.

Received: 25 June 2019 Accepted: 3 September 2019

Published online: 14 September 2019

\section{References}

1. Yoon MY, Min KB, Lee KM, Yoon Y, Kim Y, Oh YT, et al. A single gene of a commensal microbe affects host susceptibility to enteric infection. Nat Commun. 2016;7:11606

2. Hsiao A, Ahmed AM, Subramanian S, Griffin NW, Drewry LL, Petri WA Jr, et al. Members of the human gut microbiota involved in recovery from Vibrio cholerae infection. Nature. 2014;515(7527):423-6.

3. Midani FS, Weil AA, Chowdhury F, Begum YA, Khan Al, Debela MD, et al. Human gut microbiota predicts susceptibility to Vibrio cholerae infection. J Infect Dis. 2018;218(4):645-53.

4. Zhao W, Caro F, Robins W, Mekalanos JJ. Antagonism toward the intestinal microbiota and its effect on Vibrio cholerae virulence. Science. 2018; 359(6372):210-3

5. Yurist-Doutsch S, Arrieta MC, Vogt SL, Finlay BB. Gastrointestinal microbiotamediated control of enteric pathogens. Annu Rev Genet. 2014;48:361-82.

6. McKenney PT, Pamer EG. From Hype to Hope: The gut microbiota in enteric infectious disease. Cell. 2015;163(6):1326-32.

7. Kamada N, Seo SU, Chen GY, Nunez G. Role of the gut microbiota in immunity and inflammatory disease. Nat Rev Immunol. 2013;13(5):321-35.

8. Schmidt TSB, Raes J, Bork P. The human gut microbiome: from association to modulation. Cell. 2018;172(6):1198-215.

9. Anderson MC, Vonaesch P, Saffarian A, Marteyn BS, Sansonetti PJ. Shigella sonnei encodes a functional T6SS used for interbacterial competition and niche occupancy. Cell Host Microbe. 2017;21(6):769-76 e3.

10. Kommineni S, Bretl DJ, Lam V, Chakraborty R, Hayward M, Simpson P, et al. Bacteriocin production augments niche competition by enterococci in the mammalian gastrointestinal tract. Nature. 2015;526(7575):719-22.

11. Garcia-Bayona L, Comstock LE. Bacterial antagonism in host-associated microbial communities. Science. 2018;361(6408).

12. Dethlefsen $L$, Huse S, Sogin ML, Relman DA. The pervasive effects of an antibiotic on the human gut microbiota, as revealed by deep 165 rRNA sequencing. PLoS Biol. 2008:6(11):e280.

13. Russell SL, Gold MJ, Reynolds LA, Willing BP, Dimitriu P, Thorson L, et al. Perinatal antibiotic-induced shifts in gut microbiota have differential effects on inflammatory lung diseases. J Allergy Clin Immunol. 2015;135(1):100-9. 
14. Gasparrini AJ, Crofts TS, Gibson MK, Tarr PI, Warner BB, Dantas G. Antibiotic perturbation of the preterm infant gut microbiome and resistome. Gut Microbes. 2016;7(5):443-9.

15. Dethlefsen $\mathrm{L}$, Relman DA. Incomplete recovery and individualized responses of the human distal gut microbiota to repeated antibiotic perturbation. Proc Natl Acad Sci U S A. 2011;108(Suppl 1):4554-61.

16. Sprinz H, Kundel DW, Dammin GJ, Horowitz RE, Schneider H, Formal SB. The response of the germfree guinea pig to oral bacterial challenge with Escherichia coli and Shigella flexneri. Am J Pathol. 1961;39:681-95.

17. Sekirov I, Tam NM, Jogova M, Robertson ML, Li Y, Lupp C, et al. Antibioticinduced perturbations of the intestinal microbiota alter host susceptibility to enteric infection. Infect Immun. 2008;76(10):4726-36.

18. Buffie CG, Jarchum I, Equinda M, Lipuma L, Gobourne A, Viale A, et al. Profound alterations of intestinal microbiota following a single dose of clindamycin results in sustained susceptibility to Clostridium difficileinduced colitis. Infect Immun. 2012;80(1):62-73.

19. Theriot CM, Koenigsknecht MJ, Carlson PE Jr, Hatton GE, Nelson AM, Li B, et al. Antibiotic-induced shifts in the mouse gut microbiome and metabolome increase susceptibility to Clostridium difficile infection. Nat Commun. 2014:5:3114.

20. Ubeda C, Taur Y, Jenq RR, Equinda MJ, Son T, Samstein M, et al. Vancomycin-resistant Enterococcus domination of intestinal microbiota is enabled by antibiotic treatment in mice and precedes bloodstream invasion in humans. J Clin Invest. 2010;120(12):4332-41.

21. Vogt SL, Pena-Diaz J, Finlay BB. Chemical communication in the gut: Effects of microbiota-generated metabolites on gastrointestinal bacterial pathogens. Anaerobe. 2015;34:106-15.

22. Ng KM, Ferreyra JA, Higginbottom SK, Lynch JB, Kashyap PC, Gopinath S, et al. Microbiota-liberated host sugars facilitate post-antibiotic expansion of enteric pathogens. Nature. 2013;502(7469):96-9.

23. Jump RL, Polinkovsky A, Hurless K, Sitzlar B, Eckart K, Tomas M, et al. Metabolomics analysis identifies intestinal microbiota-derived biomarkers of colonization resistance in clindamycin-treated mice. PLoS One. 2014;9(7):e101267.

24. Caballero S, Kim S, Carter RA, Leiner IM, Susac B, Miller L, et al. Cooperating commensals restore colonization resistance to vancomycin-resistant Enterococcus faecium. Cell Host Microbe. 2017;21(5):592-602 e4.

25. Sekirov I, Finlay BB. The role of the intestinal microbiota in enteric infection. J Physiol. 2009;587(Pt 17):4159-67.

26. Chua K, Howden BP. Treating Gram-positive infections: vancomycin update and the whys, wherefores and evidence base for continuous infusion of anti-Gram-positive antibiotics. Curr Opin Infect Dis. 2009;22(6):525-34.

27. Hecht DW. Prevalence of antibiotic resistance in anaerobic bacteria: worrisome developments. Clin Infect Dis. 2004;39(1):92-7.

28. Lusk RH, Fekety FR Jr, Silva J Jr, Bodendorfer T, Devine BJ, Kawanishi H, et al. Gastrointestinal side effects of clindamycin and ampicillin therapy. J Infect Dis. 1977;135(Suppl):S111-9.

29. Douglas RL, Kislak JW. Treatment of Bacteroides fragilis bacteremia with clindamycin. J Infect Dis. 1973;128(4):569-71.

30. Tomioka S, Kobayashi Y. Bacteriological studies on Bacteroides fragilis infections and treatment with clindamycin for intravenous injection (author's transl). Jpn J Antibiot. 1977;30(1):30-5.

31. Hirata N, Hiramatsu K, Kishi K, Yamasaki T, Ichimiya T, Nasu M. Pretreatment of mice with clindamycin improves survival of endotoxic shock by modulating the release of inflammatory cytokines. Antimicrob Agents Chemother. 2001;45(9):2638-42.

32. Kishi K, Hirai K, Hiramatsu K, Yamasaki T, Nasu M. Clindamycin suppresses endotoxin released by ceftazidime-treated Escherichia coli O55:B5 and subsequent production of tumor necrosis factor alpha and interleukin-1 beta. Antimicrob Agents Chemother. 1999;43(3):616-22.

33. Nakano T, Hiramatsu K, Kishi K, Hirata N, Kadota J, Nasu M. Clindamycin modulates inflammatory-cytokine induction in lipopolysaccharidestimulated mouse peritoneal macrophages. Antimicrob Agents Chemother. 2003;47(1):363-7.

34. Veringa EM, Verhoef J. Clindamycin at subinhibitory concentrations enhances antibody- and complement-dependent phagocytosis by human polymorphonuclear leukocytes of Staphylococcus aureus. Chemotherapy. 1987:33(4):243-9.

35. Veringa EM, Lambe DW Jr, Ferguson DA Jr, Verhoef J. Enhancement of opsonophagocytosis of Bacteroides spp. by clindamycin in subinhibitory concentrations. J Antimicrob Chemother. 1989;23(4):577-87.

36. Sakamoto M, Benno Y. Reclassification of Bacteroides distasonis, Bacteroides goldsteinii and Bacteroides merdae as Parabacteroides distasonis gen. nov., comb. nov., Parabacteroides goldsteinii comb. nov. and Parabacteroides merdae comb. nov. Int J Syst Evol Microbiol. 2006;56(Pt 7):1599-605.

37. Song Y, Liu C, Lee J, Bolanos M, Vaisanen ML, Finegold SM. "Bacteroides goldsteinii sp. nov." isolated from clinical specimens of human intestinal origin. J Clin Microbiol. 2005;43(9):4522-7.

38. Oh YT, Park Y, Yoon MY, Bari W, Go J, Min KB, et al. Cholera toxin production during anaerobic trimethylamine $\mathrm{N}$-oxide respiration is mediated by stringent response in Vibrio cholerae. J Biol Chem. 2014; 289(19):13232-42

39. Yoon SS, Mekalanos JJ. 2,3-butanediol synthesis and the emergence of the Vibrio cholerae El Tor biotype. Infect Immun. 2006;74(12):6547-56.

40. Rhine JA, Taylor RK. TcpA pilin sequences and colonization requirements for 01 and 0139 vibrio cholerae. Mol Microbiol. 1994;13(6):1013-20.

41. Umesaki $Y$, Setoyama $H$. Immune responses of mice to orally administered asialo GM1-specific rabbit lgG in the presence or absence of cholera toxin. Immunology. 1992;75(2):386-8.

42. Sato E, Uezato T, Fujita M, Nishimura K. Developmental profiles of glycolipids in mouse small intestine. J Biochem. 1982;91(6):2013-9.

43. Arumugam $M$, Raes J, Pelletier E, Le Paslier D, Yamada T, Mende DR, et al. Enterotypes of the human gut microbiome. Nature. 2011;473:174.

44. Nagpal R, Wang S, Solberg Woods LC, Seshie O, Chung ST, Shively CA, et al. Comparative microbiome signatures and short-chain fatty acids in mouse, rat, non-human primate, and human feces. Front Microbiol. 2018:9:2897.

45. Nguyen TL, Vieira-Silva S, Liston A, Raes J. How informative is the mouse for human gut microbiota research? Dis Model Mech. 2015;8(1):1-16.

46. The Human Microbiome Project C, Huttenhower C, Gevers D, Knight R, Abubucker S, Badger JH, et al. Structure, function and diversity of the healthy human microbiome. Nature. 2012;486:207.

47. Dick LK, Bernhard AE, Brodeur TJ, Santo Domingo JW, Simpson JM, Walters SP, et al. Host distributions of uncultivated fecal Bacteroidales bacteria reveal genetic markers for fecal source identification. Appl Environ Microbiol. 2005:71(6):3184-91.

48. Lange K, Buerger M, Stallmach A, Bruns T. Effects of antibiotics on gut microbiota. Dig Dis. 2016;34(3):260-8.

49. Modi SR, Collins JJ, Relman DA. Antibiotics and the gut microbiota. J Clin Invest. 2014:124(10):4212-8.

50. Bouladoux N, Harrison OJ, Belkaid Y. The mouse model of infection with Citrobacter rodentium. Curr Protoc Immunol. 2017:119:19.5.1-.5.25.

51. Deng H, Yang S, Zhang Y, Qian K, Zhang Z, Liu Y, et al. Bacteroides fragilis prevents Clostridium difficile infection in a mouse model by restoring gut barrier and microbiome regulation. Front Microbiol. 2018;9:2976.

52. Klose KE. The suckling mouse model of cholera. Trends Microbiol. 2000;8(4):189-91.

53. Ritchie JM, Rui H, Bronson RT. Waldor MK. Back to the future: studying cholera pathogenesis using infant rabbits. mBio. 2010;1(1):e00047-10.

54. Angelichio MJ, Spector J, Waldor MK, Camilli A. Intestinal population dynamics in the suckling mouse model of infection. Infection and Immunity. 1999;67(8):3733.

55. Nygren E, Li BL, Holmgren J, Attridge SR. Establishment of an adult mouse model for direct evaluation of the efficacy of vaccines against Vibrio cholerae. Infect Immun. 2009;77(8):3475-84.

56. Sawasvirojwong S, Srimanote $P$, Chatsudthipong V, Muanprasat C. An adult mouse model of Vibrio cholerae-induced diarrhea for studying pathogenesis and potential therapy of cholera. PLoS Negl Trop Dis. 2013: 7(6):e2293.

57. Herrington DA, Hall RH, Losonsky G, Mekalanos JJ, Taylor RK, Levine MM. Toxin, toxin-coregulated pili, and the toxR regulon are essential for Vibrio cholerae pathogenesis in humans. J Exp Med. 1988;168(4):1487-92.

58. Attridge SR, Wallerstrom G, Qadri F, Svennerholm AM. Detection of antibodies to toxin-coregulated pili in sera from cholera patients. Infect Immun. 2004;72(3):1824-7.

59. Krebs SJ, Taylor RK. Protection and attachment of Vibrio cholerae mediated by the toxin-coregulated pilus in the infant mouse model. J Bacteriol. 2011; 193(19):5260-70

60. Fletcher CM, Coyne MJ, Villa OF, Chatzidaki-Livanis M, Comstock LE. A General O-Glycosylation System Important to the Physiology of a Major Human Intestinal Symbiont. Cell. 2009:137(2):321-31.

61. Hooper LV, Midtvedt T, Gordon Jl. How host-microbial interactions shape the nutrient environment of the mammalian intestine. Annu Rev Nutr. 2002;22:283-307.

62. Backhed F, Ley RE, Sonnenburg JL, Peterson DA, Gordon II. Host-bacterial mutualism in the human intestine. Science. 2005;307(5717):1915-20. 
63. Rakoff-Nahoum S, Foster KR, Comstock LE. The evolution of cooperation within the gut microbiota. Nature. 2016;533:255.

64. Sun Y, O'Riordan MXD. Regulation of bacterial pathogenesis by intestinal short-chain Fatty acids. Advances in applied microbiology. 2013;85:93-118.

65. Fu Y, Ho BT, Mekalanos JJ. Tracking Vibrio cholerae cell-cell interactions during infection reveals bacterial population dynamics within intestinal microenvironments. Cell Host and Microbe. 2018;23(2):274-81.e2.

66. Almagro-Moreno S, Boyd EF. Sialic acid catabolism confers a competitive advantage to pathogenic vibrio cholerae in the mouse intestine. Infect Immun. 2009;77(9):3807-16.

67. Maria Spagnuolo A, DiRita V, Kirschner D. A model for Vibrio cholerae colonization of the human intestine. Journal of Theoretical Biology. 2011;289:247-58.

68. Reddi G, Pruss K, Cottingham KL, Taylor RK, Almagro-Moreno S. Catabolism of mucus components influences motility of Vibrio cholerae in the presence of environmental reservoirs. PLOS ONE. 2018;13(7):e0201383.

69. McDonald ND, Lubin J-B, Chowdhury N, Boyd EF. Host-derived sialic acids are an important nutrient source required for optimal bacterial fitness in vivo. mBio. 2016;7(2):e02237-15.

70. Ramakrishna BS, Mathan VI. Colonic dysfunction in acute diarrhoea: the role of luminal short chain fatty acids. Gut. 1993;34(9):1215.

71. Baumler AJ, Sperandio V. Interactions between the microbiota and pathogenic bacteria in the gut. Nature. 2016;535(7610):85-93.

72. Patra T, Koley H, Ramamurthy T, Ghose AC, Nandy RK. The Entner-Doudoroff pathway is obligatory for gluconate utilization and contributes to the pathogenicity of Vibrio cholerae. J Bacteriol. 2012;194(13):3377-85

73. Lee KM, Park Y, Bari W, Yoon MY, Go J, Kim SC, et al. Activation of cholera toxin production by anaerobic respiration of trimethylamine $\mathrm{N}$-oxide in Vibrio cholerae. J Biol Chem. 2012;287(47):39742-52.

74. Ohashi Y, Hirayama A, Ishikawa T, Nakamura S, Shimizu K, Ueno Y, et al. Depiction of metabolome changes in histidine-starved Escherichia coli by CE-TOFMS. Mol Biosyst. 2008:4(2):135-47.

75. Ooga T, Sato H, Nagashima A, Sasaki K, Tomita M, Soga T, et al. Metabolomic anatomy of an animal model revealing homeostatic imbalances in dyslipidaemia. Mol Biosyst. 2011;7(4):1217-23.

76. Sugimoto M, Wong DT, Hirayama A, Soga T, Tomita M. Capillary electrophoresis mass spectrometry-based saliva metabolomics identified oral, breast and pancreatic cancer-specific profiles. Metabolomics. 2010; 6(1):78-95.

\section{Publisher's Note}

Springer Nature remains neutral with regard to jurisdictional claims in published maps and institutional affiliations.

Ready to submit your research? Choose BMC and benefit from:

- fast, convenient online submission

- thorough peer review by experienced researchers in your field

- rapid publication on acceptance

- support for research data, including large and complex data types

- gold Open Access which fosters wider collaboration and increased citations

- maximum visibility for your research: over $100 \mathrm{M}$ website views per year

At $\mathrm{BMC}$, research is always in progress.

Learn more biomedcentral.com/submissions 